\title{
Sheaves on Subanalytic Sites
}

\author{
Luca Prelli (*)
}

ABSTRACT - In [7] the authors introduced the notion of ind-sheaves and defined the six Grothendieck operations in this framework. As a byproduct, they obtained subanalytic sheaves and the six Grothendieck operations on them.

The aim of this paper is to give a direct construction of the six Grothendieck operations in the framework of subanalytic sites avoiding the heavy machinery of ind-sheaves. As an application, we show how to recover the subanalytic sheaves $\mathcal{O}^{t}$ and $\mathcal{O}^{\mathrm{w}}$ of temperate and Whitney holomorphic functions respectively.

\section{Introduction.}

Let $X$ be a real analytic manifold and $k$ a field. The spaces of functions which are not defined by local properties, such as tempered distributions, tempered and Whitney $\mathcal{C}^{\infty}$ functions, etc., are very useful in the study of systems of linear partial differential equations (Laplace transform, tempered holomorphic solutions of $\mathcal{D}$-modules etc.). Although these spaces do not define sheaves on $X$, they define sheaves on a site associated to $X$, the subanalytic site $X_{s a}$, where one just considers open subanalytic sets and locally finite coverings.

In [7], Kashiwara and Schapira, motivated by the construction of the microlocalization functor, treated a more general theory, namely that of ind-sheaves. They defined the category $\mathrm{I}\left(k_{X}\right)$ of ind-sheaves on $X$ as the category of ind-objects of the category $\operatorname{Mod}^{c}\left(k_{X}\right)$ of sheaves with compact support and they developped the six Grothendieck operations in this framework. When restricting to R-constructible sheaves, they showed the equivalence between the category $\mathrm{I}_{\mathbb{R}-c}\left(k_{X}\right)=\operatorname{Ind}\left(\operatorname{Mod}_{\mathbb{R}-c}^{c}\left(k_{X}\right)\right)$ of ind- $\mathbb{R}$ constructible sheaves on $X$ and the category $\operatorname{Mod}\left(k_{X_{s a}}\right)$ of sheaves on the subanalytic site associated to $X$.

(*) Indirizzo dell'A.: Dipartimento di Matematica Pura ed Applicata, Via Trieste, 63, 35121 Padova. 
In this way, tempered distributions, tempered and Whitney $\mathcal{C}^{\infty}$ functions, etc., are obtained as a byproduct of the whole theory of ind-sheaves. It turns out to be useful to have a more straightforward introduction of these sheaves.

Our aim in this paper is to give a direct, self-contained and elementary construction of the six Grothendieck operations on $\operatorname{Mod}\left(k_{X_{s a}}\right)$, without using the more sophisticated and much more difficult theory of indsheaves. Indeed, contrary to the category $\mathrm{I}\left(k_{X}\right)$, the category $\operatorname{Mod}\left(k_{X_{s a}}\right)$ is a Grothendieck category.

We will start by recalling some results of [7], the definition of a subanalytic site, the natural functor of sites $\rho: X \rightarrow X_{s a}$, and the functors $\rho_{*}$, $\rho^{-1}$ and $\rho_{\text {! }}$ relating the categories of "classical" and subanalytic sheaves. We also recall a very useful description of subanalytic sheaves as inductive limits of $\mathbb{R}$-constructible sheaves.

Then we go into the study of subanalytic sheaves, without using the notion of ind-sheaf. Let $f: X \rightarrow Y$ be a morphism of real analytic manifolds. The functors $\mathcal{H o m}, \otimes, f_{*}$ and $f^{-1}$ are well defined since $X_{s a}$ is a site. We introduce the proper direct image functor $f_{! !}$and we study the relations between the above operations and the functors $\rho_{*}, \rho^{-1}$ and $\rho_{!}$. In the derived category we obtain an exceptional inverse image, denoted by $f^{!}$, right adjoint to $R f ! !$. This is obtained via Brown representability thanks to the existence of quasi-injective objects. We study quasi-injective objects without references to ind-sheaves, we show that they are acyclic with respect to the above functors and we prove that the quasi-injective dimension of $\operatorname{Mod}\left(k_{X_{s a}}\right)$ is finite. We end this work giving some examples of subanalytic sheaves.

In more details, the contents of this paper are as follows.

In Section 1 we construct the operations in $\operatorname{Mod}\left(k_{X_{s a}}\right)$. In $\S 1.1$, we start by recalling the definitions of the functors $\rho_{*}, \rho^{-1}$ and $\rho_{!}$of [7] and their properties (a more detailed study of the functor $\rho_{\text {! }}$ is done in $\S 1.6$ ). In $\S 1.2$, we recall the internal operations and the functors of direct and inverse image (which are well defined on any site) and their relations with $\rho_{*}, \rho^{-1}$ and $\rho_{!}$. We also define (in $\S 1.4$ ) the proper direct image functor $f_{! !}$, where the notation $f_{! !}$follows from the fact that $f_{! !} \circ \rho_{*} \not \chi \rho_{*} \circ f_{!}$in general. We study its properties and the relations with the other operations. While the functors $f^{-1}$ and $\otimes$ are exact, the functors $\mathcal{H o m}, f_{*}$ and $f_{! !}$are left exact, and we introduce the subcategory of quasi-injective objects which is injective with respect to these functors. This is done in $\S 1.5$. 
In Section 2 we consider the derived category of $\operatorname{Mod}\left(k_{X_{s a}}\right)$. In $\S 2.1$, we consider the subcategory $D_{\mathrm{R}-\mathrm{c}}^{b}\left(k_{X_{s a}}\right)$ consisting of bounded complexes with $\mathbb{R}$-constructible cohomology and we prove the equivalence of derived categories $D_{\mathrm{R}-\mathrm{c}}^{b}\left(k_{X}\right) \simeq D_{\mathrm{R}-\mathrm{c}}^{b}\left(k_{X_{s a}}\right)$. Then, in $\S 2.2$, we study the derived functors of $\mathcal{H o m}, f_{*}$ and $f_{! !}$and we obtain the usual formulas (projection formula, base change formula, Künneth formula, etc.) in the framework of subanalytic sites. We also prove (in $\S 2.3$ ) some vanishing theorems for subanalytic sheaves, in particular we prove that the quasi-injective dimension of $\operatorname{Mod}\left(k_{X_{s a}}\right)$ is finite. Using the Brown representability theorem we prove the existence of a right adjoint to the functor $R f_{! !}$, denoted by $f^{!}$. This is done in $\S 2.4$. We calculate the functor $f^{!}$by decomposing $f$ as the composite of a closed embedding and a submersion.

In Section 3 we give some examples of subanalytic sheaves. We start by recalling the definition of sheaves of $\rho_{!} \mathcal{D}_{X}$-modules, where $\mathcal{D}_{X}$ denotes the sheaf of finite order differential operators on a complex analytic manifold $X$. Then in $\S 3.3$ we show how to recover the sheaves of $\rho_{!} \mathcal{D}_{X}$-modules $\mathcal{O}_{X}^{t}$ and $\mathcal{O}_{X}^{\mathrm{W}}$ of temperate and Whitney holomorphic functions of [7] respectively. We prove the relations between the above sheaves and the functors of moderate and formal cohomology of [4] and [6].

Acknowledgments. We would like to thank Prof. Pierre Schapira who encouraged us to develop a theory of subanalytic sheaves independent of that of ind-sheaves, and for his many useful remarks.

\section{Sheaves on subanalytic sites.}

In the following $X$ will be a real analytic manifold and $k$ a field. References are made to [8] and [14] for an introduction to sheaves on Grothendieck topologies, to [5] for a complete exposition on classical sheaves and R-constructible sheaves and to [1] and [10] for the theory of subanalytic sets (see also the Appendix for a short overview on some properties of subanalytic subsets).

\section{1 - The subanalytic site. Notations and review.}

We introduce the subanalytic site. The results of $\S 1.1$ have already been proved in [7], for sake of completeness we reproduce here the proofs.

Denote by $\operatorname{Op}\left(X_{s a}\right)$ the category of subanalytic subsets of $X$. One endows $\mathrm{Op}\left(X_{s a}\right)$ with the following topology: $S \subset \mathrm{Op}\left(X_{s a}\right)$ is a covering of 
$U \in \mathrm{Op}\left(X_{s a}\right)$ if for any compact subset $K$ of $X$ there exists a finite subset $S_{0}$ of $S$ such that $K \cap \bigcup_{V \in S_{0}} V=K \cap U$. We will call $X_{s a}$ the subanalytic site, and for $U \in \mathrm{Op}\left(X_{s a}\right)$ we denote by $U_{X_{s a}}$ the category $\mathrm{Op}\left(X_{s a}\right) \cap U$ with the topology induced by $X_{s a}$. There is a natural morphism of sites $U_{s a} \rightarrow U_{X_{s a}}$.

REMARK 1.1.1. We use the notation $U_{X_{s a}}$ to stress the difference from $U_{s a}$, the subanalytic site associated to $U$. For example, let $X=\mathbb{R}^{2}$ and $U=\mathbb{R}^{2} \backslash\{0\}$. Let $V_{n}=\left\{x \in \mathbb{R}^{2},|x|>\frac{1}{n}\right\}$. Then $\left\{V_{n}\right\}_{n \in \mathbb{N}} \in \operatorname{Cov}\left(U_{s a}\right)$ but $\left\{V_{n}\right\}_{n \in \mathbb{N}} \notin \operatorname{Cov}\left(U_{X_{s a}}\right)$.

Let $\operatorname{Mod}\left(k_{X_{s a}}\right)$ denote the category of sheaves on $X_{s a}$. Then $\operatorname{Mod}\left(k_{X_{s a}}\right)$ is a Grothendieck category, i.e. it admits a generator and small inductive limits, and small filtrant inductive limits are exact. In particular as a Grothendieck category, $\operatorname{Mod}\left(k_{X_{s a}}\right)$ has enough injective objects.

REMARK 1.1.2. Denote by $\mathrm{Op}^{c}\left(X_{s a}\right)$ the category of relatively compact subanalytic open subsets of $X$. One denotes by $X_{s a}^{c}$ the category $\operatorname{Op}^{c}\left(X_{s a}\right)$ with the topology induced by $X_{s a}$. The forgetful functor gives an equivalence of categories $\operatorname{Mod}\left(k_{X_{s a}}\right) \stackrel{\sim}{\rightarrow} \operatorname{Mod}\left(k_{X_{s a}^{c}}\right)$.

Proposition 1.1.3. Let $\left\{F_{i}\right\}_{i \in I}$ be a filtrant inductive system in $\operatorname{Mod}\left(k_{X_{s a}}\right)$ and let $U \in \operatorname{Op}^{c}\left(X_{s a}\right)$. Then

$$
\underset{i}{\lim } \Gamma\left(U ; F_{i}\right) \stackrel{\sim}{\rightarrow} \Gamma\left(U ; \underset{i}{\lim } F_{i}\right) .
$$

Proof. By Remark 1.1.2 it is enough to prove the assertion in the category $\operatorname{Mod}\left(k_{X_{s a}^{c}}\right)$. Denote by "lim" $\underset{i}{\longrightarrow} F_{i}$ the presheaf $V \mapsto \underset{i}{\lim } \Gamma\left(V ; F_{i}\right)$ on $X_{s a}^{c}$. Let $U \in \operatorname{Op}^{c}\left(X_{s a}\right)$ and let $S$ be a finite covering of $U$. Since lim commutes with finite projective limits we obtain the isomorphism $\left(\underset{i}{\stackrel{\text { "lim" }}{\longrightarrow}} F_{i}\right)(S) \underset{i}{\stackrel{\sim}{\rightarrow}} \underset{i}{\lim } F_{i}(S)$ and $F_{i}(U) \stackrel{\sim}{\rightarrow} F_{i}(S)$ since $F_{i} \in \operatorname{Mod}\left(k_{X_{s a}^{c}}\right)$ for each $i$. Moreover the family of finite coverings of $U$ is cofinal in $\operatorname{Cov}(U)$. Hence $\underset{i}{\stackrel{\lim }{\longrightarrow}} F_{i} \stackrel{\sim}{\stackrel{(}{\rightarrow}}\left(\underset{i}{\longrightarrow} F_{i}\right)^{+}$. Applying once again the functor $(\cdot)^{+}$we get

$$
\underset{i}{\stackrel{\lim }{\longrightarrow}} F_{i} \simeq\left(\underset{i}{\stackrel{\lim }{\longrightarrow}} F_{i}\right)^{+} \simeq\left(\underset{i}{\stackrel{\lim }{\longrightarrow}} F_{i}\right)^{++} \simeq \underset{i}{\lim } F_{i} .
$$

Hence applying the functor $\Gamma(U ; \cdot)$ we obtain the isomorphism $\underset{i}{\lim } \Gamma\left(U ; F_{i}\right) \stackrel{\sim}{\rightarrow} \Gamma\left(U ; \underset{i}{\lim } F_{i}\right)$ for each $U \in \mathrm{Op}^{c}\left(X_{s a}\right)$. 
There is an easy way to construct sheaves on a subanalytic site

Proposition 1.1.4. Let $F$ be a presheaf on $X_{s a}^{c}$ and assume that

(i) $F(\varnothing)=0$,

(ii) For any $U, V \in \mathrm{Op}^{c}\left(X_{s a}\right)$ the sequence $0 \rightarrow F(U \cup V) \rightarrow$ $\rightarrow F(U) \oplus F(V) \rightarrow F(U \cap V)$ is exact.

Then $F \in \operatorname{Mod}\left(k_{X_{s a}^{c}}\right) \simeq \operatorname{Mod}\left(k_{X_{s a}}\right)$.

Proof. Let $U \in \mathrm{Op}^{c}\left(X_{s a}\right)$ and let $\left\{U_{j}\right\}_{j=1}^{n}$ be a finite covering of $U$. Set for short $U_{i j}=U_{i} \cap U_{j}$. We have to show the exactness of the sequence

$$
0 \rightarrow F(U) \rightarrow \oplus_{1 \leq k \leq n} F\left(U_{k}\right) \rightarrow \oplus_{1 \leq i<j \leq n} F\left(U_{i j}\right),
$$

where the second morphism sends $\left(s_{k}\right)_{1 \leq k \leq n}$ to $\left(t_{i j}\right)_{1 \leq i<j \leq n}$ by $t_{i j}=$ $=\left.s_{i}\right|_{U_{i j}}-\left.s_{j}\right|_{U_{i j}}$. We shall argue by induction on $n$. For $n=1$ the result is trivial, and $n=2$ is the hypothesis. Suppose that the assertion is true for $j \leq n-1$ and set $U^{\prime}=\bigcup_{1 \leq k<n} U_{k}$. By the induction hypothesis the following commutative diagram is exact

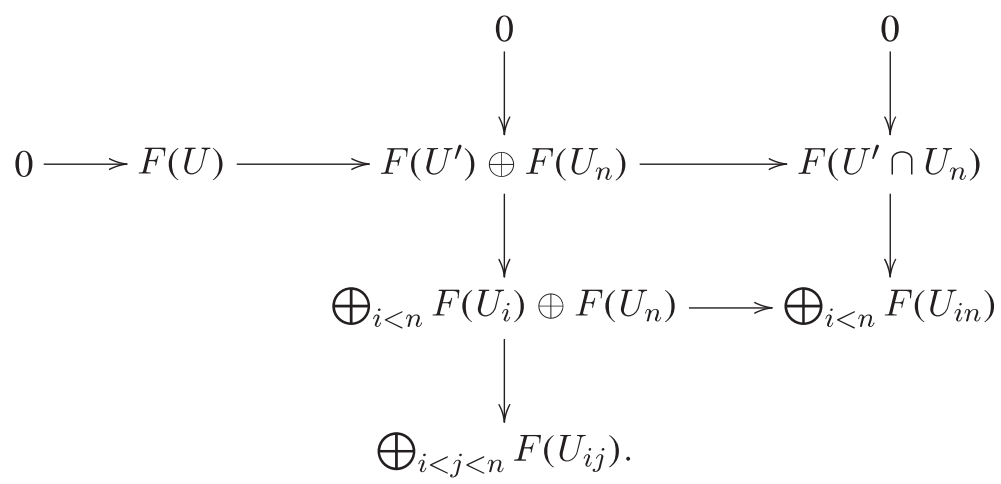

Then the result follows.

Let $\operatorname{Mod}_{\mathbb{R}-c}\left(k_{X}\right)$ be the abelian category of $\mathbb{R}$-constructible sheaves on $X$, and consider its subcategory $\operatorname{Mod}_{\mathbb{R}-c}^{c}\left(k_{X}\right)$ consisting of sheaves whose support is compact.

We denote by

$$
\rho: X \rightarrow X_{s a}
$$


the natural morphism of sites. We have functors

$$
\operatorname{Mod}_{\mathbb{R}-\mathrm{c}}^{c}\left(k_{X}\right) \subset \operatorname{Mod}_{\mathrm{R}-\mathrm{c}}\left(k_{X}\right) \subset \operatorname{Mod}\left(k_{X}\right) \underset{\rho^{-1}}{\stackrel{\rho_{*}}{\rightleftarrows}} \operatorname{Mod}\left(k_{X_{s a}}\right) .
$$

We will still denote by $\rho_{*}$ the restriction of $\rho_{*}$ to $\operatorname{Mod}_{\mathbb{R}-\mathrm{c}}\left(k_{X}\right)$ and $\operatorname{Mod}_{\mathbb{R}-\mathrm{c}}^{c}\left(k_{X}\right)$.

REMark 1.1.5. By Proposition 1.1.3 for each $F \in \operatorname{Mod}\left(k_{X}\right)$ and $V \in \mathrm{Op}^{c}\left(X_{s a}\right)$ one has

$$
\Gamma\left(V ; \rho_{*} F\right) \simeq \underset{U}{\lim } \Gamma\left(V ; \rho_{*} F_{U}\right) \simeq \Gamma\left(V ; \underset{U}{\lim } \rho_{*} F_{U}\right),
$$

where $U$ ranges through the family of relatively compact open subanalytic subsets of $X$. The first isomorphism follows since $V$ is relatively compact and $\Gamma\left(V, \rho_{*} F_{U}\right) \simeq \Gamma\left(V, \rho_{*} F\right)$ if $V \subset U$. The isomorphism (1.2) implies $\underset{U}{\lim } \rho_{*} F_{U} \stackrel{\sim}{\rightarrow} \rho_{*} F$.

REMARK 1.1.6. The functor $\rho_{*}$ does not commute with filtrant inductive limits. For example consider the family $\left\{V_{n}\right\}_{n \in \mathbb{N}}$ of Remark 1.1.1. We have $\rho_{*} \underset{n}{\lim } k_{V_{n}} \simeq \rho_{*} k_{\mathbb{R}^{2} \backslash\{0\}}$, while for each $U \in \mathrm{Op}^{c}\left(\mathbb{R}_{s a}^{2}\right)$ with $0 \in \partial U$ we have $\Gamma\left(U ; \underset{n}{\lim } \rho_{*} k_{V_{n}}\right) \simeq \underset{n}{\lim } \Gamma\left(U ; \rho_{*} k_{V_{n}}\right)=0$.

Proposition 1.1.7. Let $U$ be an open subanalytic subset of $X$ and consider the constant sheaf $k_{U_{X_{s a}}} \in \operatorname{Mod}\left(k_{X_{s a}}\right)$. We have $k_{U_{X_{s a}}} \simeq \rho_{*} k_{U}$.

Proof. Let $F$ be the presheaf on $X_{s a}$ defined by $F(V)=k$ if $V \subset U$, $F(V)=0$ otherwise. This is a separated presheaf and $k_{U_{X_{s a}}}=F^{++}$. Moreover there is an injective arrow $F(V) \hookrightarrow \rho_{*} k_{U}(V)$ for each $V \in \mathrm{Op}\left(X_{s a}\right)$. Hence $F^{++} \hookrightarrow \rho_{*} k_{U}$ since the functor $(\cdot)^{++}$is exact. Let $\mathcal{T}$ be the family of $W \in \mathrm{Op}\left(X_{s a}\right)$ connected and such that $W$ does not contain any connected component of $U$. Then $\mathcal{T}$ forms a basis for the topology of $X_{s a}$ since the connected components of $U$ are locally finite. For each $W \in \mathcal{T}$ we have $F(W) \simeq \rho_{*} k_{U}(W) \simeq k \quad$ if $\quad W \subset U$ and $F(W)=0$ otherwise. Then $F^{++} \simeq \rho_{*} k_{U}$.

Proposition 1.1.8. The restriction of $\rho_{*}$ to $\operatorname{Mod}_{\mathbb{R}-\mathrm{c}}\left(k_{X}\right)$ is exact.

Proof. (i) Let us consider an epimorphism $G \rightarrow F$ in $\operatorname{Mod}_{\mathrm{R}-\mathrm{c}}^{c}\left(k_{X}\right)$, we have to prove that $\psi: \rho_{*} G \rightarrow \rho_{*} F$ is an epimorphism. Let 
$U \in \mathrm{Op}^{c}\left(X_{s a}\right) \quad$ and let $\quad 0 \neq s \in \Gamma\left(U ; \rho_{*} F\right) \simeq \operatorname{Hom}_{k_{X}}\left(k_{U}, F\right)$. Set $G^{\prime}=G \times_{F} k_{U}$. Then $G^{\prime} \in \operatorname{Mod}_{\mathrm{R}-\mathrm{c}}^{c}\left(k_{X}\right)$ and moreover $G^{\prime} \rightarrow k_{U}$. There exists a finite $\left\{U_{i}\right\}_{i \in I} \subset \mathrm{Op}^{c}\left(X_{s a}\right)$ with $U_{i}$ connected for each $i$ such that $\oplus_{i} k_{U_{i}} \rightarrow G^{\prime}$. The composition $k_{U_{i}} \rightarrow G^{\prime} \rightarrow k_{U}$ is given by the multiplication by $a_{i} \in k$. Set $I_{0}=\left\{k_{U_{i}} ; a_{i} \neq 0\right\}$, we may assume $a_{i}=1$. We get a diagram

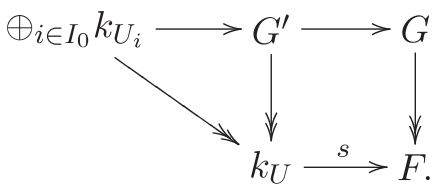

The composition $k_{U_{i}} \rightarrow G^{\prime} \rightarrow G$ defines $t_{i} \in \operatorname{Hom}_{k_{X}}\left(k_{U_{i}}, G\right)=\Gamma\left(U_{i} ; \rho_{*} G\right)$. Hence for each $s \in \Gamma\left(U ; \rho_{*} F\right)$ there exists a finite covering $\left\{U_{i}\right\}$ of $U$ and $t_{i} \in \Gamma\left(U_{i} ; \rho_{*} G\right)$ such that $\psi\left(t_{i}\right)=\left.s\right|_{U_{i}}$. This means that $\psi$ is surjective.

(ii) Let $F \in \operatorname{Mod}_{\mathbb{R}-\mathrm{c}}\left(k_{X}\right)$. By Remark 1.1.5 $\rho_{*} F \simeq \underset{U}{\lim } \rho_{*} F_{U}$, where $U$ ranges through the family $\mathrm{Op}^{c}\left(X_{s a}\right)$. The result follows since $\rho_{*}$ is exact on $\operatorname{Mod}_{\mathrm{R}-\mathrm{c}}^{c}\left(k_{X}\right)$ and filtrant $\underline{\lim }$ are exact.

Proposition 1.1.9. The restriction of $\rho_{*}$ to $\operatorname{Mod}_{\mathbb{R}-c}\left(k_{X}\right)$ is fully faithful.

Proof. It is enough to prove that $\rho^{-1} \rho_{*} F \simeq F$ for each $F \in \operatorname{Mod}_{\mathbb{R}-c}\left(k_{X}\right)$. Since both functors are exact on $\operatorname{Mod}_{\mathbb{R}-c}\left(k_{X}\right)$ we may reduce to the case $F=k_{U}$ with $U \in \mathrm{Op}\left(X_{s a}\right)$ and the result follows from Proposition 1.1.7.

Notations 1.1.10. Since the functor $\rho_{*}$ is fully faithful and exact on the category $\operatorname{Mod}_{\mathbb{R}-\mathrm{c}}\left(k_{X}\right)$, we can identify $\operatorname{Mod}_{\mathrm{R}-\mathrm{c}}\left(k_{X}\right)$ with its image in $\operatorname{Mod}\left(k_{X_{s a}}\right)$. When there is no risk of confusion we will write $F$ instead of $\rho_{*} F$, for $F \in \operatorname{Mod}_{\mathbb{R}-\mathrm{c}}\left(k_{X}\right)$.

The following theorem gives a fundamental characterization of subanalytic sheaves and it will be used systematically in the following Sections.

THEOREM 1.1.11. (i) Let $G \in \operatorname{Mod}_{\mathbb{R}-\mathrm{c}}^{c}\left(k_{X}\right)$ and let $\left\{F_{i}\right\}$ be a filtrant inductive system in $\operatorname{Mod}\left(k_{X_{s a}}\right)$. Then we have an isomorphism

$$
\underset{i}{\lim } \operatorname{Hom}_{k_{X_{s a}}}\left(\rho_{*} G, F_{i}\right) \stackrel{\sim}{\rightarrow} \operatorname{Hom}_{k_{X_{s a}}}\left(\rho_{*} G, \underset{i}{\lim } F_{i}\right) .
$$


(ii) Let $F \in \operatorname{Mod}\left(k_{X_{s a}}\right)$. There exists a small filtrant inductive system $\left\{F_{i}\right\}_{i \in I}$ in $\operatorname{Mod}_{\mathbb{R}-c}^{c}\left(k_{X}\right)$ such that $F \simeq \underset{i}{\lim } \rho_{*} F_{i}$.

Proof. (i) There exists an exact sequence $G_{1} \rightarrow G_{0} \rightarrow G \rightarrow 0$ with $G_{1}, G_{0}$ finite direct sums of constant sheaves $k_{U}$ with $U \in \mathrm{Op}^{c}\left(X_{s a}\right)$. Since $\rho_{*}$ is exact on $\operatorname{Mod}_{\mathbb{R}-c}\left(k_{X}\right)$ and commutes with finite sums, by Proposition 1.1.7 we are reduced to prove the isomorphism $\underset{i}{\lim } \Gamma\left(U ; F_{i}\right) \stackrel{\sim}{\rightarrow} \Gamma\left(U ; \underset{i}{\lim } F_{i}\right)$. Then the result follows from Proposition 1.1.3.

(ii) Let $F \in \operatorname{Mod}\left(k_{X_{s a}}\right)$, and define

$$
\begin{aligned}
I_{0} & :=\left\{(U, s) ; U \in \mathrm{Op}^{c}\left(X_{s a}\right), s \in \Gamma(U ; F)\right\} \\
G_{0} & :=\oplus_{(U, s) \in I_{0}} \rho_{*} k_{U}
\end{aligned}
$$

The morphism $\rho_{*} k_{U} \rightarrow F$, where the section $1 \in \Gamma\left(U ; k_{U}\right)$ is sent to $s \in \Gamma(U ; F)$ defines un epimorphism $\varphi: G_{0} \rightarrow F$. Replacing $F$ by $\operatorname{ker} \varphi$ we construct a sheaf $G_{1}=\oplus_{(V, t) \in I_{1}} \rho_{*} k_{V}$ and an epimorphism $G_{1} \rightarrow \operatorname{ker} \varphi$. Hence we get an exact sequence $G_{1} \rightarrow G_{0} \rightarrow F \rightarrow 0$. For $J_{0} \subset I_{0}$ set for short $G_{J_{0}}=\oplus_{(U, s) \in J_{0}} \rho_{*} k_{U}$ and define similarly $G_{J_{1}}$. Set

$$
J=\left\{\left(J_{1}, J_{0}\right) ; J_{k} \subset I_{k}, J_{k} \text { is finite and }\left.\operatorname{im} \varphi\right|_{G_{J_{1}}} \subset G_{J_{0}}\right\} .
$$

The category $J$ is filtrant and $F \simeq \underset{\left(J_{1}, J_{0}\right) \in J}{\lim } \operatorname{coker}\left(G_{J_{1}} \rightarrow G_{J_{0}}\right)$.

Proposition 1.1.12. Let $F \in \operatorname{Mod}\left(k_{X_{s a}}\right)$, and let $U \in \mathrm{Op}(X)$. Then

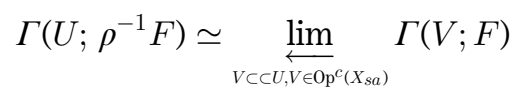

Proof. By Theorem 1.1.11 we may assume $F=\underset{i}{\lim } \rho_{*} F_{i}$, with $F_{i} \in \operatorname{Mod}_{\mathrm{R}-\mathrm{c}}^{c}\left(k_{X}\right)$. Then $\rho^{-1} F \simeq \underset{i}{\lim } \rho^{-1} \rho_{*} F_{i} \simeq \underset{i}{\lim } F_{i}$. We have the chain of
isomorphisms

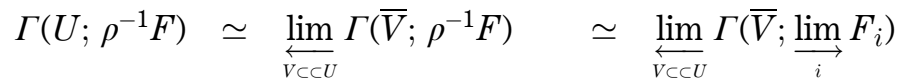

$$
\begin{aligned}
& \simeq \lim _{V \subset \subset U} \underset{i}{\lim } \Gamma\left(\bar{V} ; F_{i}\right) \simeq \lim _{V \subset \subset U} \underset{i}{\lim } \Gamma\left(V ; F_{i}\right) \\
& \simeq \lim _{V \subset \subset U} \underset{i}{\lim } \Gamma\left(V ; \rho_{*} F_{i}\right) \simeq \lim _{V \subset \subset U} \Gamma(V ; F)
\end{aligned}
$$

where $V \in \mathrm{Op}^{c}\left(X_{s a}\right)$. The third isomorphism follows since $\bar{V}$ is compact and the last isomorphism follows from Proposition 1.1.3. 
Proposition 1.1.13. One has $\rho^{-1} \circ \rho_{*} \stackrel{\sim}{\rightarrow} \mathrm{id}$, in particular the functor $\rho_{*}$ is fully faithful.

Proof. Let $F \in \operatorname{Mod}\left(k_{X}\right)$. Every $x \in X$ has a fundamental neighborhood system consisting of open subanalytic subsets. Hence we have the chain of isomorphisms

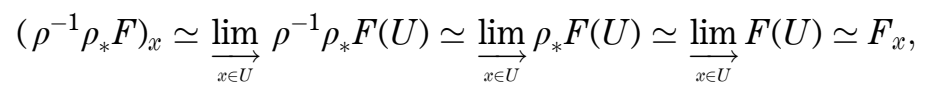

where $U$ ranges through the family of open subanalytic neighborhoods of $x$. The second isomorphism follows since, by Proposition 1.1.12

$$
\underset{x \in U}{\lim _{x \rightarrow}} \rho^{-1} \rho_{*} F(U) \simeq \underset{x \in U}{\lim _{V \subset \subset U}} \lim _{*} F(V) \simeq \underset{x \in U}{\lim _{\longrightarrow}} \rho_{*} F(U)
$$

Now we will describe a left adjoint to the functor $\rho^{-1}$.

Proposition 1.1.14. The functor $\rho^{-1}$ admits a left adjoint, denoted by $\rho_{!}$. It satisfies

(i) for $F \in \operatorname{Mod}\left(k_{X}\right)$ and $U \in \operatorname{Op}\left(X_{s a}\right), \rho_{!} F$ is the sheaf associated to the presheaf $U \mapsto \Gamma(\bar{U} ; F)$,

(ii) for $U \in \mathrm{Op}(X)$ one has $\rho_{!} k_{U} \simeq \underset{V \subset \subset U, V \in \mathrm{lp}^{c}\left(X_{s a}\right)}{\lim _{V}} k_{V}$.

Proof. Let $\widetilde{F} \in \operatorname{Psh}\left(k_{X_{s a}}\right)$ be the presheaf $U \mapsto \Gamma(\bar{U} ; F)$, and let $G \in \operatorname{Mod}\left(k_{X_{s a}}\right)$. We will construct morphisms

$$
\operatorname{Hom}_{P s h\left(k_{X_{s} a}\right)}(\widetilde{F}, G) \underset{\vartheta}{\stackrel{\xi}{\rightleftarrows}} \operatorname{Hom}_{k_{X}}\left(F, \rho^{-1} G\right) .
$$

To define $\xi$, let $\varphi: \widetilde{F} \rightarrow G$ and $U \in \mathrm{Op}(X)$. Then the morphism $\xi(\varphi)(U): F(U) \rightarrow \rho^{-1} G(U)$ is defined as follows

$$
F(U) \simeq \lim _{V \subset \subset U, V \in 0^{c}{ }^{c}\left(X_{s a}\right)} F(\bar{V}) \stackrel{\varphi}{\longrightarrow} \lim _{V \subset \subset U, V \in 0^{c}\left(X_{S a}\right)} G(V) \simeq \rho^{-1} G(U) .
$$

On the other hand, let $\psi: F \rightarrow \rho^{-1} G$ and $U \in \mathrm{Op}^{c}\left(X_{s a}\right)$. Then the morphism $\vartheta(\psi)(U): \widetilde{F}(U) \rightarrow G(U)$ is defined as follows

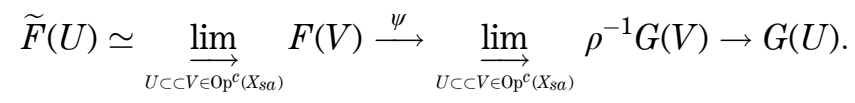


By construction one can check that the morphisms $\xi$ and $\vartheta$ are inverse to each others. Then (i) follows from the chain of isomorphisms

$$
\operatorname{Hom}_{k_{X_{s a}}}\left(\widetilde{F}^{++}, G\right) \simeq \operatorname{Hom}_{P s h\left(k_{X_{s a}}\right)}(\widetilde{F}, G) \simeq \operatorname{Hom}_{k_{X_{s a}}}\left(F, \rho^{-1} G\right) .
$$

To show (ii), consider the following sequence of isomorphisms

$$
\begin{aligned}
\operatorname{Hom}_{k_{X_{s a}}}\left(\rho_{!} k_{U}, F\right) & \simeq \operatorname{Hom}_{k_{X}}\left(k_{U}, \rho^{-1} F\right) \\
& \simeq \underbrace{}_{V \subset \subset U, V \in 0_{\mathrm{p}} c\left(X_{s a}\right)} \operatorname{Hom}_{k_{X_{s a}}}\left(k_{V}, F\right) \\
& \simeq \operatorname{Hom}_{k_{X_{s a}}}(\underbrace{}_{V \subset \subset U, \overrightarrow{V \in O p} c^{c}\left(X_{s a}\right)} k_{V}, F),
\end{aligned}
$$

where the second isomorphism follows from Proposition 1.1.12.

Proposition 1.1.15. The functor $\rho_{!}$is exact and commutes with $\mathrm{lim}$ and $\otimes$.

Proof. It follows by adjunction that $\rho_{\text {! }}$ is right exact and commutes with lim, so let us show that it is also left exact. With the notations of Proposition 1.1.14, let $F \in \operatorname{Mod}\left(k_{X}\right)$, and let $\widetilde{F} \in \operatorname{Psh}\left(k_{X_{s a}}\right)$ be the presheaf $U \mapsto \Gamma(\bar{U} ; F)$. Then $\rho_{!} F \simeq \widetilde{F}^{++}$, and the functors $F \mapsto \widetilde{F}$ and $G \mapsto G^{++}$are left exact.

Let us show that $\rho_{!}$commutes with $\otimes$. Let $F, G \in \operatorname{Mod}\left(k_{X}\right)$, the morphism

$$
F(\bar{U}) \otimes G(\bar{U}) \rightarrow(F \otimes G)(\bar{U})
$$

defines a morphism in $\operatorname{Mod}\left(k_{X_{s a}}\right)$

$$
\rho_{!} F \otimes \rho_{!} G \rightarrow \rho_{!}(F \otimes G)
$$

by Proposition 1.1.14 (i). Since $\rho_{\text {! }}$ commutes with lim we may suppose that $F=k_{U}$ and $G=k_{V}$ and the result follows from Proposition 1.1.14 (ii).

Proposition 1.1.16. The functor $\rho_{!}$is fully faithful. In particular one has $\rho^{-1} \circ \rho_{!} \simeq$ id. Moreover, for $F \in \operatorname{Mod}\left(k_{X}\right)$ and $G \in \operatorname{Mod}\left(k_{X_{s a}}\right)$ one has

$$
\rho^{-1} \mathcal{H o m}\left(\rho_{!} F, G\right) \simeq \mathcal{H o m}\left(F, \rho^{-1} G\right) .
$$

Proof. For $F, G \in \operatorname{Mod}\left(k_{X}\right)$ we have by adjunction

$$
\operatorname{Hom}_{k_{X}}\left(\rho^{-1} \rho_{!} F, G\right) \simeq \operatorname{Hom}_{k_{X}}\left(F, \rho^{-1} \rho_{*} G\right) \simeq \operatorname{Hom}_{k_{X}}(F, G) .
$$


This also implies that $\rho_{!}$is fully faithful, in fact

$$
\operatorname{Hom}_{k_{X_{s a}}}\left(\rho_{!} F, \rho_{!} G\right) \simeq \operatorname{Hom}_{k_{X}}\left(F, \rho^{-1} \rho_{!} G\right) \simeq \operatorname{Hom}_{k_{X}}(F, G) .
$$

Now let $K, F \in \operatorname{Mod}\left(k_{X}\right)$ and $G \in \operatorname{Mod}\left(k_{X_{s a}}\right)$, we have

$$
\begin{aligned}
\operatorname{Hom}_{k_{X}}\left(K, \rho^{-1} \mathcal{H o m}\left(\rho_{!} F, G\right)\right) & \simeq \operatorname{Hom}_{k_{X_{s a}}}\left(\rho_{!} K, \mathcal{H o m}\left(\rho_{!} F, G\right)\right) \\
& \simeq \operatorname{Hom}_{k_{X_{s a}}}\left(\rho_{!} K \otimes \rho_{!} F, G\right) \\
& \simeq \operatorname{Hom}_{k_{X_{s a}}}\left(\rho_{!}(K \otimes F), G\right) \\
& \simeq \operatorname{Hom}_{k_{X}}\left(K \otimes F, \rho^{-1} G\right) \\
& \simeq \operatorname{Hom}_{k_{X}}\left(K, \mathcal{H o m}\left(F, \rho^{-1} G\right)\right)
\end{aligned}
$$

and the result follows.

1.2 - Operations on the subanalytic site.

Let $X, Y$ be two real analytic manifolds, and let $f: X \rightarrow Y$ be a real analytic map. This defines a morphism of sites $f: X_{s a} \rightarrow Y_{s a}$. We have a diagram

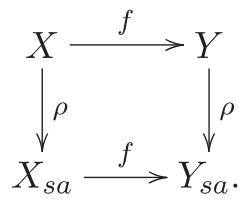

The following functors are always well defined on a site

$$
\begin{aligned}
& \mathcal{H} o m: \operatorname{Mod}\left(k_{X_{s a}}\right)^{o p} \times \operatorname{Mod}\left(k_{X_{s a}}\right) \rightarrow \operatorname{Mod}\left(k_{X_{s a}}\right), \\
& \otimes: \operatorname{Mod}\left(k_{X_{s a}}^{o p}\right) \times \operatorname{Mod}\left(k_{X_{s a}}\right) \rightarrow \operatorname{Mod}\left(k_{X_{s a}}\right), \\
& f_{*}: \operatorname{Mod}\left(k_{X_{s a}}\right) \rightarrow \operatorname{Mod}\left(k_{Y_{s a}}\right), \\
& f^{-1}: \operatorname{Mod}\left(k_{Y_{s a}}\right) \rightarrow \operatorname{Mod}\left(k_{X_{s a}}\right) .
\end{aligned}
$$

Let us summarize their properties:

- the functor $\mathcal{H o m}$ is left exact and commutes with $\rho_{*}$,

- the functor $\otimes$ is exact and commutes with $\underline{\lim }, \rho^{-1}$ and $\rho_{!}$,

- the functor $f_{*}$ is left exact and commutes with $\rho_{*}$ and $\underset{\longleftarrow}{\longleftarrow}$,

- the functor $f^{-1}$ is exact and commutes with $\underline{\lim }, \otimes$ and $\rho^{-1}$,

- $\left(f^{-1}, f_{*}\right)$ is a pair of adjoint functors. 
Let $Z$ be a subanalytic locally closed subset of $X$. As in classical sheaf theory we define

$$
\begin{aligned}
\Gamma_{Z}: \operatorname{Mod}\left(k_{X_{s a}}\right) & \rightarrow \operatorname{Mod}\left(k_{X_{s a}}\right) \\
F & \mapsto \mathcal{H o m}\left(\rho_{*} k_{Z}, F\right) \\
(\cdot)_{Z}: \operatorname{Mod}\left(k_{X_{s a}}\right) & \rightarrow \operatorname{Mod}\left(k_{X_{s a}}\right) \\
F & \mapsto F \otimes \rho_{*} k_{Z} .
\end{aligned}
$$

We have

- the functor $\Gamma_{Z}$ is left exact and commutes with $\rho_{*}$ and $\lim$,

- the functor $(\cdot)_{Z}$ is exact and commutes with $\underline{\lim }, \otimes$ and $\rho^{-1}$,

- $\left((\cdot)_{Z}, \Gamma_{Z}\right)$ is a pair of adjoint functors.

\section{3 - R-constructible sheaves on subanalytic sites.}

Let us consider the category $\operatorname{Mod}_{\mathrm{R}-\mathrm{c}}\left(k_{X}\right)$. We prove that the subcategory of $\operatorname{Mod}\left(k_{X_{s a}}\right)$ consisting of $\mathbb{R}$-constructible sheaves is stable under inverse image and tensor product.

Proposition 1.3.1. Let $F, G \in \operatorname{Mod}_{\mathrm{R}-\mathrm{c}}\left(k_{X}\right)$. Then $\rho_{*}(F \otimes G) \simeq \rho_{*} F \otimes$ $\otimes \rho_{*} G$.

Proof. We may reduce to the case $F=k_{U}, G=k_{V}$ with $U, V \in$ $\mathrm{Op}\left(X_{s a}\right)$. In this case $\rho_{*} k_{U \cap V} \simeq \rho_{*} k_{U} \otimes \rho_{*} k_{V}$ by Proposition 1.1.7.

Corollary 1.3.2. Let $F \in \operatorname{Mod}_{\mathbb{R}-\mathrm{c}}\left(k_{X}\right)$, and let $Z$ be a subanalytic locally closed subset of $X$. Then $\rho_{*} F_{Z} \simeq\left(\rho_{*} F\right)_{Z}$.

Let $X, Y$ be two real analytic manifolds, and let $f: X \rightarrow Y$ be a real analytic map.

Proposition 1.3.3. Let $f: X \rightarrow Y$ be a real analytic map. Let $G \in$ $\operatorname{Mod}_{\mathrm{R}-\mathrm{c}}\left(k_{Y}\right)$. Then $\rho_{*} f^{-1} G \simeq f^{-1} \rho_{*} G$.

Proof. Since the functor $f^{-1}$ is exact, we may reduce to the case $G=k_{V}$, with $V \in \mathrm{Op}\left(Y_{\text {sa }}\right)$. In this case we have $\rho_{*} f^{-1} k_{V} \simeq \rho_{*} k_{f^{-1}(V)} \simeq$ $\simeq f^{-1} \rho_{*} k_{V}$, where the last isomorphism follows from Proposition 1.1.7.

We apply the above results to calculate the functor Hom in the category $\operatorname{Mod}\left(k_{X_{s a}}\right)$. 
Proposition 1.3.4. Let $F=\underline{\lim _{\longrightarrow}} F_{i}$, with $F_{i} \in \operatorname{Mod}\left(k_{X_{s a}}\right)$ and let $G=\underset{j}{\lim _{*}} \rho_{*} G_{j}$ with $G_{j} \in \operatorname{Mod}_{\mathbb{R}-\mathrm{c}}\left(k_{X}\right)$. One has

$$
\mathcal{H o m}(G, F) \simeq \lim _{j} \underset{i}{\lim } \mathcal{H o m}\left(\rho_{*} G_{j}, F_{i}\right) .
$$

Proof. For each $U \in \mathrm{Op}^{c}\left(X_{s a}\right)$ one has the isomorphisms

$$
\begin{aligned}
& \Gamma(U, \mathcal{H o m}(G, F)) \simeq \operatorname{Hom}_{k_{X_{s u}}}\left(G_{U}, F\right) \\
& \simeq \lim _{j} \underset{i}{\lim _{i}} \operatorname{Hom}_{k_{X_{s a}}}\left(\rho_{*} G_{j U}, F_{i}\right) \\
& \simeq \varliminf_{j} \underset{i}{\lim } \Gamma\left(U ; \mathcal{H o m}\left(\rho_{*} G_{j}, F_{i}\right)\right) \\
& \simeq \Gamma\left(U ; \underset{j}{\lim _{i}} \underset{\lim _{i}}{\longrightarrow} \mathcal{H o m}\left(\rho_{*} G_{j}, F_{i}\right)\right) .
\end{aligned}
$$

In the second isomorphism we used Corollary 1.3.2, and the last isomorphism follows from Proposition 1.1.3 and because $\Gamma(U ; \cdot)$ commutes with $\lim _{\longleftarrow}$.

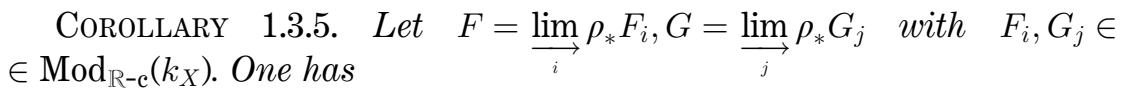

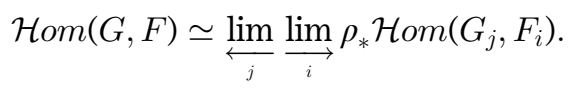

Proof. It follows from the fact that $\mathcal{H o m}$ commutes with $\rho_{*}$ and from Proposition 1.3.4.

COROLlary 1.3.6. Let $F=\underline{\lim } \rho_{*} F_{i}$, with $F_{i} \in \operatorname{Mod}_{\mathbb{R}-\mathrm{c}}^{c}(X)$ be a sheaf on $X_{\text {sa }}$. Let $Z$ be a subanalytic locally closed subset of $X$. Then $\Gamma_{Z} F \simeq \underset{\rightarrow}{\lim } \rho_{*} \Gamma_{Z} F_{i}$.

\section{4 - Proper direct image on $\operatorname{Mod}\left(k_{X_{s a}}\right)$.}

In [7] the authors defined the functor $f_{! !}$of proper direct image using ind-sheaves. Here we give a direct construction:

$$
\begin{aligned}
& f_{! !}: \operatorname{Mod}\left(k_{X_{s a}}\right) \rightarrow \operatorname{Mod}\left(k_{Y_{s a}}\right)
\end{aligned}
$$

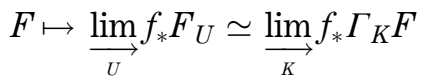

where $U$ ranges through the family of relatively compact open subanalytic 
subsets of $X$ and $K$ ranges through the family of subanalytic compact subsets of $X$. One shall be aware that $\lim$ is taken in the category $\operatorname{Mod}\left(k_{Y_{s a}}\right)$. Let $V \in \mathrm{Op}^{c}\left(Y_{s a}\right)$. Then $\left.\Gamma \overrightarrow{\left(V ; f_{!} F\right.}\right)=\underset{U}{\lim } \Gamma\left(f^{-1}(V) ; F_{U}\right) \simeq$ $\underset{K}{\lim } \Gamma\left(f^{-1}(V) ; \Gamma_{K} F\right)$, where $U$ ranges through the family of relatively compact open subanalytic subsets of $X$ and $K$ ranges through the family of subanalytic compact subsets of $X$. If $f$ is proper on $\operatorname{supp}(F)$ then $f_{*} \simeq f_{! !}$and in this case $f_{! !} \circ \rho_{*} \simeq \rho_{*} \circ f_{!}$.

REMARK 1.4.1. Remark that $f_{! !} \circ \rho_{*} \neq \rho_{*} \circ f_{!}$in general. Indeed let $V \in \mathrm{Op}^{c}\left(Y_{s a}\right)$, then

$$
\begin{aligned}
& \Gamma\left(V ; f_{! !} \rho_{*} F\right)=\underset{\mathrm{K}}{\lim } \Gamma\left(f^{-1}(V) ; \Gamma_{K} F\right), \\
& \Gamma\left(V ; \rho_{*} f_{!} F\right)=\underset{Z}{\longrightarrow} \Gamma\left(f^{-1}(V) ; \Gamma_{Z} F\right),
\end{aligned}
$$

where $Z$ ranges through the family of closed subsets of $f^{-1}(V)$ such that $\left.f\right|_{Z}: Z \rightarrow V$ is proper. Then

$$
\begin{aligned}
& \Gamma\left(V ; f_{! !} \rho_{*} F\right)=\left\{s \in \Gamma\left(f^{-1}(V) ; F\right) ; \overline{\operatorname{supp}(s)} \text { is compact in } X\right\}, \\
& \Gamma\left(V ; \rho_{*} f_{!} F\right)=\left\{s \in \Gamma\left(f^{-1}(V) ; F\right) ; f: \operatorname{supp}(s) \rightarrow V \text { is proper }\right\} .
\end{aligned}
$$

For example, let $f: \mathbb{R}^{2} \rightarrow \mathbb{R}$ be the projection on the first coordinate, and let $V=(a, b) \in \mathrm{Op}^{c}\left(\mathbb{R}_{s a}\right)$. Suppose that $\operatorname{supp}(s)=\{(x, y) \in(a, b) \times \mathbb{R}$, $\left.y=\frac{1}{(x-a)(b-x)}\right\}$. Then $f: \operatorname{supp}(s) \rightarrow V$ is proper but $\overline{\operatorname{supp}(s)}$ is not compact.

Proposition 1.4.2. The functor $f_{! !}$commutes with filtrant $\mathrm{lim}$. Moreover $\rho^{-1} \circ f_{! !} \simeq f_{!} \circ \rho^{-1}$.

Proof. Let us show that $f_{! !}$commutes with filtrant lim. Let $V \in \operatorname{Op}^{c}\left(Y_{s a}\right)$ and let $\left\{F_{i}\right\}_{i}$ be a filtrant inductive system in $\operatorname{Mod}\left(k_{X_{s a}}\right)$. Then

$$
\begin{aligned}
\underset{K}{\lim _{\longrightarrow}} \operatorname{Hom}_{k_{X_{s a}}}\left(k_{f^{-1}(V)}, \Gamma_{K} \underset{i}{\left.\lim _{i} F_{i}\right)}\right. & \simeq \underset{K}{\lim } \operatorname{Hom}_{k_{X_{s a}}}\left(k_{f^{-1}(V) \cap K}, \underset{i}{\lim } F_{i}\right) \\
& \simeq \underset{i, K}{\lim } \operatorname{Hom}_{k_{X_{s a}}}\left(k_{f^{-1}(V) \cap K}, F_{i}\right) \\
& \simeq \underset{i, K}{\lim } \operatorname{Hom}_{k_{X_{s a}}}\left(k_{f^{-1}(V)}, \Gamma_{K} F_{i}\right) \\
& \simeq \underset{i}{\lim } \operatorname{Hom}_{k_{Y_{s a}}}\left(k_{V}, f_{! !} F_{i}\right) \\
& \simeq \operatorname{Hom}_{k_{Y_{s a}}}\left(k_{V}, \underset{i}{\left.\lim f_{! !} F_{i}\right),}\right.
\end{aligned}
$$


where the second isomorphism follows from the fact that $k_{f^{-1}(V) \cap K} \in$ $\in \operatorname{Mod}_{\mathrm{R}-\mathrm{c}}^{c}\left(k_{X}\right)$.

Let us show $\rho^{-1} \circ f_{! !} \simeq f_{!} \circ \rho^{-1}$. Let $F=\underset{i}{\lim _{\longrightarrow} \rho_{*}} F_{i}$. Since $f_{! !}$commutes with filtrant $\stackrel{\lim }{\longrightarrow}$ and $F_{i}$ has compact support for each $i$ we have $f_{! !} F=\underset{\lim }{\longrightarrow} \rho_{*} f_{!} F_{i}$. We have the chain of isomorphisms

$$
\begin{aligned}
f_{!} \rho^{-1} \underset{i}{\lim _{*}} \rho_{*} F_{i} & \simeq f_{!} \underset{i}{\lim _{\longrightarrow}} \rho^{-1} \rho_{*} F_{i} \simeq f_{!} \underset{i}{\lim _{\longrightarrow}} F_{i} \simeq \underset{i}{\lim } f_{!} F_{i} \\
& \simeq \underset{i}{\lim } \rho^{-1} \rho_{*} f_{!} F_{i} \simeq \rho^{-1} \underset{i}{\underset{\lim }{\longrightarrow}} \rho_{*} f_{!} F_{i} .
\end{aligned}
$$

Proposition 1.4.3. The functor $f_{*}$ commutes with $\rho^{-1}$.

Proof. Let $F \in \operatorname{Mod}\left(k_{X_{s a}}\right)$. Then $f_{*} F \simeq \underset{\lim _{K}}{\lim } f_{*} F_{K}$, where $K$ ranges through the family of subanalytic compact subsets of $X$. We have the chain of isomorphisms

$$
\begin{aligned}
& f_{*} \rho^{-1} F \simeq \longleftarrow_{K}^{\lim } f_{*}\left(\rho^{-1} F\right)_{K} \simeq \overleftarrow{\lim }_{K} f_{! !}\left(\rho^{-1} F\right)_{K} \simeq \overleftarrow{\lim }_{K} f_{! !} \rho^{-1} F_{K} \\
& \simeq \underbrace{\lim }_{K} \rho^{-1} f_{!} F_{K} \simeq \rho^{-1}{\underset{\lim }{K}}_{f_{!}} F_{K} \simeq \rho^{-1} \overleftarrow{K}_{\lim _{*}} f_{*} F_{K} \simeq \rho^{-1} f_{*} F,
\end{aligned}
$$

where the second and the sixth isomorphism follow from the fact that $f$ is proper on a compact subset of $X$.

Corollary 1.4.4. The functor $f^{-1}$ commutes with $\rho_{!}$.

PROOF. It follows immediately by adjunction.

Proposition 1.4.5. Let $F \in \operatorname{Mod}\left(k_{X_{s a}}\right)$ and $G \in \operatorname{Mod}\left(k_{Y_{s a}}\right)$. Then

$$
f_{! !} F \otimes G \simeq f_{! !}\left(F \otimes f^{-1} G\right) \text {. }
$$

Proof. Let $F=\underset{i}{\lim } \rho_{*} F_{i}, G=\underset{j}{\lim } \rho_{*} G_{j}$. The functors $\otimes, f_{! !}$and $f^{-1}$ commute with $\underset{i}{\lim }$. Moreover $\operatorname{supp}\left(F_{i} \otimes f^{-1} G_{j}\right)$ is compact for each $i, j$, 
hence $f$ is proper on it. Then

$$
\begin{aligned}
f_{! !} \underset{i}{\lim } \rho_{*} F_{i} \otimes \underset{j}{\lim } \rho_{*} G_{j} & \simeq \underset{i, j}{\lim } \rho_{*}\left(f_{!} F_{i} \otimes G_{j}\right) \\
& \simeq \underset{i, j}{\lim } \rho_{*}\left(f_{!}\left(F_{i} \otimes f^{-1} G_{j}\right)\right) \\
& \simeq f_{! !}\left(\underset{i}{\lim } \rho_{*} F_{i} \otimes f^{-1} \underset{i}{\lim } \rho_{*} G_{j}\right) .
\end{aligned}
$$

In the first isomorphism we used Proposition 1.3.1 and in the last one we used Propositions 1.3.1 and 1.3.3.

Now let us consider a cartesian square

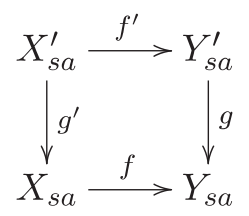

Proposition 1.4.6. Let $F \in \operatorname{Mod}\left(k_{X_{s a}}\right)$. Then $g^{-1} f_{! !} F \simeq f_{! !}^{\prime} g^{\prime-1} F$.

Proof. Let $F=\underset{i}{\lim } \rho_{*} F_{i}$. All the functors in the above formula commute with $\underset{i}{\lim }$. Moreover since $\operatorname{supp}\left(F_{i}\right)$ is compact, $f^{\prime}$ is proper on $\operatorname{supp}\left(g^{\prime-1} F_{i}\right)$ for each $i$. Then

$$
g^{-1} f_{! !} \underset{i}{\lim } \rho_{*} F_{i} \simeq \underset{i}{\lim } \rho_{*} g^{-1} f_{!} F_{i} \simeq \underset{i}{\lim } \rho_{*} f_{!}^{\prime} g^{\prime-1} F_{i} \simeq f_{! !}^{\prime} g^{\prime-1} \underset{i}{\lim } \rho_{*} F_{i},
$$

where the first and the last isomorphisms follow from Proposition 1.3.3.

The following isomorphism is the analogue for subanalytic sheaves of Corollary 4.3.15 of [7].

Proposition 1.4.7. Let $G \in \operatorname{Mod}_{\mathrm{R}-\mathrm{c}}\left(k_{Y}\right)$ and let $F \in \operatorname{Mod}\left(k_{X_{s a}}\right)$. Then the natural morphism

$$
f_{! !} \mathcal{H o m}\left(f^{-1} G, F\right) \rightarrow \mathcal{H o m}\left(G, f_{! !} F\right)
$$

is an isomorphism.

Proof. Let us construct the morphism. By adjunction we have

$$
f^{-1} G \otimes \mathcal{H o m}\left(f^{-1} G, F\right) \rightarrow F,
$$


hence, using the projection formula we get

$$
G \otimes f_{! !} \mathcal{H o m}\left(f^{-1} G, F\right) \simeq f_{! !}\left(f^{-1} G \otimes \mathcal{H o m}\left(f^{-1} G, F\right)\right) \rightarrow f_{! !} F,
$$

then by adjunction we obtain the desired morphism. Let us show that it is an isomorphism. We have the chain of isomorphisms

$$
\begin{aligned}
& f ! \operatorname{Hom}\left(f^{-1} G, F\right) \simeq \underset{K}{\lim _{K}} f_{*} \Gamma_{K} \mathcal{H o m}\left(f^{-1} G, F\right) \\
& \simeq \underset{K}{\lim } f_{*} \mathcal{H o m}\left(f^{-1} G, \Gamma_{K} F\right) \\
& \simeq \underset{K}{\lim } \operatorname{Hom}\left(G, f_{*} \Gamma_{K} F\right) \\
& \simeq \mathcal{H o m}\left(G, \underset{K}{\lim _{\longrightarrow}} f_{*} \Gamma_{K} F\right) \\
& \simeq \mathcal{H o m}\left(G, f_{! !} F\right),
\end{aligned}
$$

where the fourth isomorphism follows from Proposition 1.3.4.

\section{5 - Quasi-injective objects.}

Let us introduce a category which is useful in order to find acyclic objects with respect to the functors defined in the previous Sections. Although the definition is inspired to the definition of quasi-injective objects of [7] (or, more generally, to the definition of quasi-injective objects of a ind-category, see [8] for more details), the proofs of Theorems 1.5.4 and 1.5.16 are independent of the theory of ind-objects.

Definition 1.5.1. An object $F \in \operatorname{Mod}\left(k_{X_{s a}}\right)$ is quasi-injective if the functor $\operatorname{Hom}_{k_{X_{s u}}}(\cdot, F)$ is exact in $\operatorname{Mod}_{\mathrm{R}-\mathrm{c}}^{c}\left(k_{X}\right)$ or, equivalently (see Theorem 8.7.2 of [8]) if for each $U, V \in \mathrm{Op}^{c}\left(X_{s a}\right)$ with $V \subset U$ the restriction morphism $\Gamma(U ; F) \rightarrow \Gamma(V ; F)$ is surjective.

It follows from the definition that injective sheaves belong to $\mathcal{J}_{X_{s a}}$. This implies that $\mathcal{J}_{X_{s a}}$ is cogenerating. Moreover the category $\mathcal{J}_{X_{s a}}$ is stable by filtrant lim and $\prod$.

Proposition 1.5.2. Let $0 \rightarrow F^{\prime} \rightarrow F \rightarrow F^{\prime \prime} \rightarrow 0$ be an exact sequence in $\operatorname{Mod}\left(k_{X_{s a}}\right)$ and assume that $F^{\prime}$ is quasi-injective. Let $U \in \operatorname{Op}^{c}\left(X_{s a}\right)$. 
Then the sequence

$$
0 \rightarrow \Gamma\left(U ; F^{\prime}\right) \rightarrow \Gamma(U ; F) \rightarrow \Gamma\left(U ; F^{\prime \prime}\right) \rightarrow 0
$$

is exact.

Proof. Let $s^{\prime \prime} \in \Gamma\left(U ; F^{\prime \prime}\right)$, and let $\left\{V_{i}\right\}_{i=1}^{n}$ be a finite covering of $U$ such that there exists $s_{i} \in \Gamma\left(V_{i} ; F\right)$ whose image is $\left.s^{\prime \prime}\right|_{V_{i}}$. For $n \geq 2$ on $V_{1} \cap V_{2} s_{1}-s_{2}$ defines a section of $\Gamma\left(V_{1} \cap V_{2} ; F^{\prime}\right)$ which extends to $s^{\prime} \in \Gamma\left(U ; F^{\prime}\right)$. Replace $s_{1}$ with $s_{1}-s^{\prime}$. We may suppose that $s_{1}=s_{2}$ on $V_{1} \cap V_{2}$. Then there exists $t \in \Gamma\left(V_{1} \cup V_{2}\right)$ such that $\left.t\right|_{V_{i}}=s_{i}, i=1,2$. Thus the induction proceeds.

Proposition 1.5.3. Let $F^{\prime}, F, F^{\prime \prime} \in \operatorname{Mod}\left(k_{X_{s a}}\right)$, and consider the exact sequence

$$
0 \rightarrow F^{\prime} \rightarrow F \rightarrow F^{\prime \prime} \rightarrow 0 .
$$

Suppose that $F^{\prime}, F \in \mathcal{J}_{X_{s a}}$. Then $F^{\prime \prime} \in \mathcal{J}_{X_{s a}}$.

Proof. Let $U, V \in \mathrm{Op}^{c}\left(X_{s a}\right)$ with $V \subset U$ and let us consider the diagram below

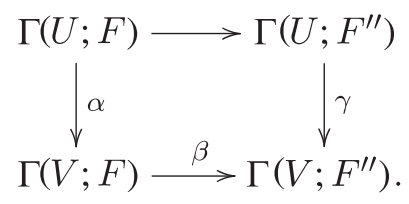

The morphism $\alpha$ is surjective since $F$ is quasi-injective and $\beta$ is surjective by Proposition 1.5.2. Then $\gamma$ is surjective.

THEOREM 1.5.4. The family of quasi-injective sheaves is injective with respect to the functor $\operatorname{Hom}_{k_{X_{s a}}}(G, \cdot)$ for each $G \in \operatorname{Mod}_{\mathbb{R}-\mathrm{c}}\left(k_{X}\right)$.

Proof. (i) Let $0 \rightarrow F^{\prime} \rightarrow F \rightarrow F^{\prime \prime} \rightarrow 0$ be an exact sequence in $\operatorname{Mod}\left(k_{X_{s a}}\right)$ and assume that $F^{\prime} \in \mathcal{J}_{X_{s a}}$. Let $G \in \operatorname{Mod}_{\mathbb{R}-c}^{c}\left(k_{X}\right)$. We have to show that the sequence

$$
0 \rightarrow \operatorname{Hom}_{k_{X_{s u}}}\left(G, F^{\prime}\right) \rightarrow \operatorname{Hom}_{k_{X_{s u}}}(G, F) \rightarrow \operatorname{Hom}_{k_{X_{s a}}}\left(G, F^{\prime \prime}\right) \rightarrow 0
$$

is exact. There is an epimorphism $\varphi: \oplus_{i \in I} k_{U_{i}} \rightarrow G$ where $I$ is finite and $U_{i} \in \mathrm{Op}^{c}\left(X_{s a}\right)$ for each $i \in I$.

The sequence $0 \rightarrow \operatorname{ker} \varphi \rightarrow \oplus_{i \in I} k_{U_{i}} \rightarrow G \rightarrow 0$ is exact. We set for short $G_{1}=\operatorname{ker} \varphi$ and $G_{2}=\oplus_{i \in I} k_{U_{i}}$. We get the following diagram where the first 
column is exact

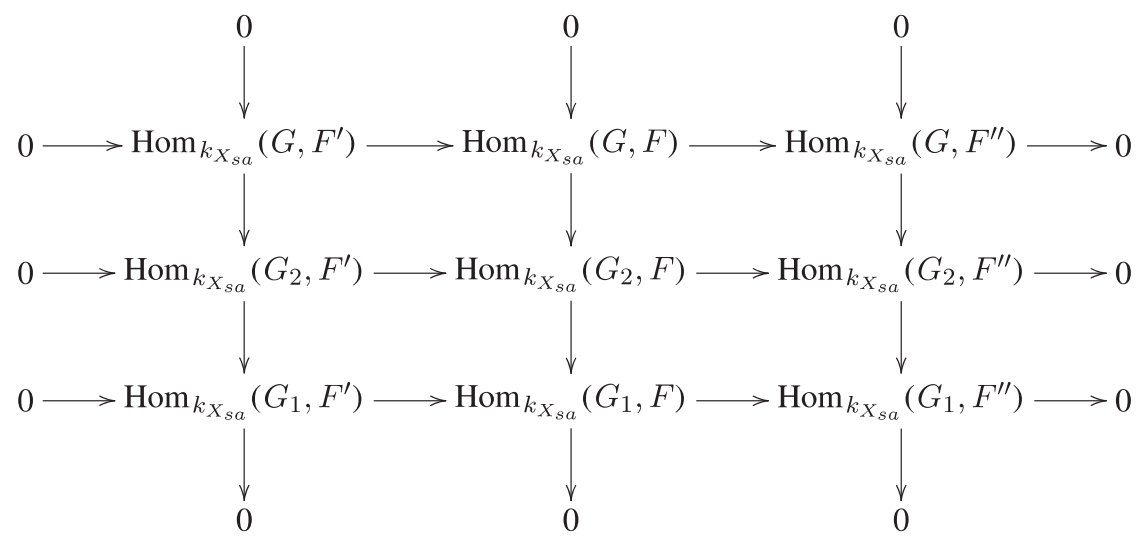

The second row is exact by Proposition 1.5.2, hence the top row is exact by the snake lemma.

(ii) Let $G \in \operatorname{Mod}_{\mathbb{R}-c}\left(k_{X}\right)$, let $0 \rightarrow F^{\prime} \rightarrow F \rightarrow F^{\prime \prime} \rightarrow 0$ be an exact sequence in $\operatorname{Mod}\left(k_{X_{s a}}\right)$ with $F^{\prime} \in \mathcal{J}_{X_{s a}}$. Let $\left\{V_{n}\right\}_{n \in \mathbb{N}} \in \operatorname{Cov}\left(X_{s a}\right)$ such that $V_{n} \subset \subset V_{n+1}$. By (i), all the sequences

$$
0 \rightarrow \operatorname{Hom}_{k_{X_{s a}}}\left(G_{V_{n}}, F^{\prime}\right) \rightarrow \operatorname{Hom}_{k_{X_{s u}}}\left(G_{V_{n}}, F\right) \rightarrow \operatorname{Hom}_{k_{X_{s u}}}\left(G_{V_{n}}, F^{\prime \prime}\right) \rightarrow 0
$$

are exact. Moreover since $F^{\prime} \in \mathcal{J}_{X_{s a}}$ the morphism $\operatorname{Hom}_{k_{X_{s a}}}\left(G_{V_{n+1}}, F^{\prime}\right) \rightarrow$ $\rightarrow \operatorname{Hom}_{k_{X_{s a}}}\left(G_{V_{n}}, F^{\prime}\right)$ is surjective for all $n$. Then by the Mittag-Leffler property (see Proposition 1.12.3 of [5]) the sequence

$0 \rightarrow \underbrace{\lim }_{n} \operatorname{Hom}_{k_{X_{s a}}}\left(G_{V_{n}}, F^{\prime}\right) \rightarrow \underset{n}{\lim } \operatorname{Hom}_{k_{X_{s a}}}\left(G_{V_{n}}, F\right) \rightarrow \underbrace{\lim }_{n} \operatorname{Hom}_{k_{X_{s a}}}\left(G_{V_{n}}, F^{\prime \prime}\right) \rightarrow 0$

is exact. Since ${\underset{n}{n}}_{\lim _{n}} \operatorname{Hom}_{k_{X_{s a}}}\left(G_{V_{n}}, \cdot\right) \simeq \operatorname{Hom}_{k_{X_{s a}}}(G, \cdot)$ the result follows.

Proposition 1.5.5. Let $G \in \operatorname{Mod}_{\mathbb{R}-\mathrm{c}}\left(k_{X}\right)$. Then quasi-injective sheaves are injective with respect to the functor $\mathcal{H o m}(G, \cdot)$.

Proof. Let $G \in \operatorname{Mod}_{\mathbb{R}-c}\left(k_{X}\right)$. It is enough to check that for each $U \in \mathrm{Op}\left(X_{s a}\right)$ and each exact sequence $0 \rightarrow F^{\prime} \rightarrow F \rightarrow F^{\prime \prime} \rightarrow 0$ with $F^{\prime} \in \mathcal{J}_{X_{s a}}$, the sequence

$$
0 \rightarrow \Gamma\left(U ; \mathcal{H o m}\left(G, F^{\prime}\right)\right) \rightarrow \Gamma(U ; \mathcal{H o m}(G, F)) \rightarrow \Gamma\left(U ; \mathcal{H o m}\left(G, F^{\prime \prime}\right)\right) \rightarrow 0
$$

is exact. We have $\Gamma(U, \mathcal{H o m}(G, \cdot)) \simeq \operatorname{Hom}_{k_{x_{s a}}}\left(G_{U}, \cdot\right)$, and quasi-injective objects are injective with respect to the functor $\operatorname{Hom}_{k_{X_{s a}}}\left(G_{U}, \cdot\right)$ for each $G \in \operatorname{Mod}_{\mathrm{R}-\mathrm{c}}\left(k_{X}\right)$, and for each $U \in \mathrm{Op}\left(X_{s a}\right)$. 
COROLLARY 1.5.6. Quasi-injective sheaves are injective with respect to the functor $\Gamma_{Z}$ for each locally closed subanalytic subset $Z$ of $X$.

Corollary 1.5.7. Let $F \in \operatorname{Mod}\left(k_{X_{s a}}\right)$ be quasi-injective. Then the functor $\operatorname{Hom}(\cdot, F)$ is exact on $\operatorname{Mod}_{\mathbb{R}-c}\left(k_{X}\right)$.

Proof. Let $F \in \operatorname{Mod}\left(k_{X_{s a}}\right)$ be quasi-injective. There is an isomorphism of functors $\Gamma(U ; \mathcal{H o m}(\cdot, F)) \simeq \operatorname{Hom}_{k_{X_{s a}}}\left((\cdot)_{U}, F\right)$ for each $U \in \mathrm{Op}\left(X_{s a}\right)$. The functor $\operatorname{Hom}_{k_{X_{s a}}}\left((\cdot)_{U}, F\right)$ is exact on $\operatorname{Mod}_{\mathbb{R}-c}\left(k_{X}\right)$ and the result follows.

Proposition 1.5.8. Let $F \in \operatorname{Mod}\left(k_{X_{s a}}\right)$. Then $F$ is quasi-injective if and only if $\mathcal{H o m}(G, F)$ is quasi-injective for each $G \in \operatorname{Mod}_{\mathbb{R}-c}\left(k_{X}\right)$.

Proof. (i) Let $F$ be quasi-injective, and let $G \in \operatorname{Mod}_{\mathbb{R}-c}\left(k_{X}\right)$. We have $\operatorname{Hom}_{k_{X_{s a}}}(\cdot, \mathcal{H o m}(G, F)) \simeq \operatorname{Hom}_{k_{X_{s a}}}(\cdot \otimes G, F)$, and $\operatorname{Hom}_{k_{X_{s a}}}(\cdot \otimes G, F)$ is exact on $\operatorname{Mod}_{\mathrm{R}-\mathrm{c}}^{c}\left(k_{X}\right)$.

(ii) Suppose that $\mathcal{H o m}(G, F)$ is quasi-injective for each $G \in \operatorname{Mod}_{\mathbb{R}-c}\left(k_{X}\right)$. The result follows by setting $G=k_{X}$.

COROLlaRY 1.5.9. The functor $\Gamma_{Z}$ sends quasi-injective objects to quasi-injective objects for each locally closed subanalytic subset $Z$ of $X$.

Let $f: X \rightarrow Y$ be a morphism of real analytic manifolds.

PROPOSITION 1.5.10. Quasi-injective sheaves are injective with respect to the functor $f_{*}$. The functor $f_{*}$ sends quasi-injective objects to quasiinjective objects.

Proof. (i) Let us consider $V \in \mathrm{Op}\left(Y_{s a}\right)$. There is an isomorphism of functors $\Gamma\left(V ; f_{*}(\cdot)\right) \simeq \Gamma\left(f^{-1}(V) ; \cdot\right)$. It follows from Proposition 1.5.4 that $\mathcal{J}_{X_{s a}}$ is injective with respect to the functor $\Gamma\left(f^{-1}(V) ; \cdot\right) \simeq$ $\simeq \operatorname{Hom}_{k_{X_{s a}}}\left(k_{f^{-1}(V)}, \cdot\right)$ for any $V \in \mathrm{Op}\left(Y_{s a}\right)$.

(ii) Let $F \in \mathcal{J}_{X_{s a}}$. For each $G \in \operatorname{Mod}_{\mathbb{R}-c}^{c}\left(k_{Y}\right)$ we have $\operatorname{Hom}_{k_{Y_{s a}}}\left(G, f_{*} F\right) \simeq$ $\operatorname{Hom}_{k_{X_{s}}}\left(f^{-1} G, F\right)$. Since $f^{-1}$ is exact and sends $\operatorname{Mod}_{\mathrm{R}-\mathrm{c}}^{\mathrm{c}}\left(k_{Y}\right)$ to $\operatorname{Mod}_{\mathrm{R}-\mathrm{c}}\left(k_{X}\right)$, Proposition 1.5.4 implies that the functor $\operatorname{Hom}_{k_{X_{s a}}}\left(f^{-1}(\cdot), F\right)$ is exact on $\operatorname{Mod}_{\mathbb{R}-\mathrm{c}}^{c}\left(k_{Y}\right)$.

Proposition 1.5.11. The family of quasi-injective sheaves is $f_{! !}$-injective. The functor $f_{! !}$sends quasi-injective objects to quasi-injective objects. 
Proof. (i) Let $0 \rightarrow F^{\prime} \rightarrow F \rightarrow F^{\prime \prime} \rightarrow 0$ be an exact sequence in $\operatorname{Mod}\left(k_{X_{s a}}\right)$ and assume that $F^{\prime} \in \mathcal{J}_{X_{s a}}$. We have to check that the sequence $0 \rightarrow f_{! !} F^{\prime} \rightarrow f_{! !} F \rightarrow f_{! !} F^{\prime \prime} \rightarrow 0$ is exact. Since $F^{\prime} \in \mathcal{J}_{X_{s a}}$, we have $\Gamma_{K} F^{\prime} \in \mathcal{J}_{X_{s a}}$. Moreover $\mathcal{J}_{X_{s a}}$ is injective with respect to $\Gamma_{K}$ and $f_{*}$. This implies that the sequence

$$
0 \rightarrow f_{*} \Gamma_{K} F^{\prime} \rightarrow f_{*} \Gamma_{K} F \rightarrow f_{*} \Gamma_{K} F^{\prime \prime} \rightarrow 0
$$

is exact. Applying the exact functor $\underset{K}{\lim }$ we find that the sequence

$$
0 \rightarrow \underset{K}{\lim } f_{*} \Gamma_{K} F^{\prime} \rightarrow \underset{K}{\lim } f_{*} \Gamma_{K} F \rightarrow \underset{K}{\lim } f_{*} \Gamma_{K} F^{\prime \prime} \rightarrow 0
$$

is exact.

(ii) Let $K$ be a compact subanalytic subset of $X$. The functors $\Gamma_{K}$ and $f_{*}$ send quasi-injective objects to quasi-injective objects, then $f_{*} \Gamma_{K} F \in \mathcal{J}_{Y_{s a}}$. Since $\mathcal{J}_{Y_{s a}}$ is stable by filtrant $\underline{\mathrm{lim}}$, the result follows.

Let $S$ be a closed subanalytic subset of $X$ and let $i_{S}: S \hookrightarrow X$ be the closed embedding. Let $F=\underset{i}{\lim } \rho_{*} F_{i} \in \operatorname{Mod}\left(k_{X_{s a}}\right)$ with $F_{i} \in \operatorname{Mod}_{\mathrm{R}-\mathrm{c}}^{c}\left(k_{X}\right)$. We have $F_{S} \simeq \underset{i}{\lim } \rho_{*} F_{i S} \simeq \underset{i}{\lim } \rho_{*} i_{S *} i_{S}{ }^{-1} F_{i} \simeq i_{S *} i_{S}{ }^{-1} F$.

LEMMa 1.5.12. Let $S$ be a closed subanalytic subset of $X$ and let $U \in \mathrm{Op}^{c}\left(X_{s a}\right)$. Let $F \in \operatorname{Mod}\left(k_{X_{s a}}\right)$. Then $\Gamma\left(U ; F_{S}\right) \simeq \underset{V \supset \supset \cap \cap}{\lim } \Gamma(V ; F)$, with
$V \in \operatorname{Op}^{c}\left(X_{s a}\right)$.

Proof. Let $F \in \operatorname{Mod}\left(k_{X_{s a}}\right)$. Then $F \simeq \underset{i}{\lim } \rho_{*} F_{i}$ with $F_{i} \in \operatorname{Mod}_{\mathrm{R}-\mathrm{c}}^{c}\left(k_{X}\right)$. We have the chain of isomorphisms

$$
\begin{aligned}
\Gamma\left(U ; F_{S}\right) & \simeq \underset{i}{\lim } \Gamma\left(U ; F_{i S}\right) \\
& \simeq \underset{i, V \supset S \cap U}{\lim } \Gamma\left(V ; F_{i}\right) \\
& \simeq \underset{V \supset S \cap U}{\lim } \Gamma(V ; F),
\end{aligned}
$$

where $V$ ranges through the family of relatively compact open subanalytic subsets of $X$ containing $S \cap U$. The second isomorphism follows since $F_{i}$ is $\mathbb{R}$-constructible for each $i$.

REMARK 1.5.13. The fact that $F_{i} \in \operatorname{Mod}_{\mathrm{R}-\mathrm{c}}\left(k_{X}\right)$ is locally constant on a subanalytic stratification of $X$ plays an essential role. In fact the iso- 
morphism is not true in general in $\operatorname{Mod}\left(k_{X}\right)$, since the family of relatively compact open subanalytic subsets of $X$ containing $S \cap U$ is not cofinal to the family of open subsets of $X$ containing $S \cap U$.

Proposition 1.5.14. Let $S$ be a closed subanalytic subset of $X$ and let $F \in \operatorname{Mod}\left(k_{X_{s a}}\right)$ be quasi-injective. Then $F_{S}$ is quasi-injective.

Proof. Let $U, V \in \mathrm{Op}^{c}\left(X_{s a}\right)$ with $V \subset U$. Since $F$ is quasi-injective and inductive limits are right exact, the morphism $\underset{U^{\prime} \supset S \cap U}{\lim } \Gamma\left(U^{\prime} ; F\right) \rightarrow$ $\lim _{\longrightarrow} \Gamma\left(V^{\prime} ; F\right)$ with $V^{\prime}, U^{\prime} \in \mathrm{Op}^{c}\left(X_{s a}\right)$, is surjective. Hence by Lemma 1.5.12 $\underset{V^{\prime} \supset \cap \cap V}{\longrightarrow}$ the morphism $\Gamma\left(U ; F_{S}\right) \rightarrow \Gamma\left(V ; F_{S}\right)$ is surjective and the result follows.

Recall that $F \in \operatorname{Mod}\left(k_{X}\right)$ is c-soft if the natural morphism $\Gamma(X ; F) \rightarrow$ $\Gamma(K, F)$ is surjective for each compact $K \subset X$. If $F$ is c-soft and $Z$ is a locally closed subset of $X$, then $F_{Z}$ is c-soft. Moreover c-soft sheaves are $\Gamma(U ; \cdot)$-injective for each $U \in \mathrm{Op}(X)$.

Proposition 1.5.15. Let $F \in \operatorname{Mod}\left(k_{X_{s a}}\right)$ be quasi-injective. Then $\rho^{-1} F$ is c-soft.

Proof. Let $K$ be a compact subset of $X$. Recall that if $U \in \mathrm{Op}(X)$ then $\Gamma\left(U ; \rho^{-1} F\right) \simeq \lim _{V \subset \subset U} \Gamma(V ; F)$, where $V \in \mathrm{Op}\left(X_{s a}\right)$. We have the chain of isomorphisms

$$
\begin{aligned}
\Gamma\left(K ; \rho^{-1} F\right) & \simeq \underset{U}{\lim } \Gamma\left(U ; \rho^{-1} F\right) \\
& \simeq \underset{U}{\lim } \underset{V \subset \subset U}{\lim } \Gamma(V ; F) \\
& \simeq \underset{U}{\lim } \Gamma(U ; F)
\end{aligned}
$$

where $U$ ranges through the family of subanalytic relatively compact open subsets of $X$ containing $K$ and $V \in \mathrm{Op}\left(X_{s a}\right)$.

Since $F$ is quasi-injective and filtrant inductive limits are exact, the morphism $\Gamma\left(X ; \rho^{-1} F\right) \simeq \Gamma(X ; F) \rightarrow \underset{U}{\lim } \Gamma(U ; F) \simeq \Gamma\left(K ; \rho^{-1} F\right)$, where $U$ ranges through the family of subanalytic open subsets of $X$ containing $K$, is surjective. 
Let us consider the following subcategory of $\operatorname{Mod}\left(k_{X_{s a}}\right)$ : $\mathcal{P}_{X_{s a}}:=\left\{G \in \operatorname{Mod}\left(k_{X_{s a}}\right) ; G\right.$ is $\operatorname{Hom}_{k_{X s a}}(\cdot, F)$-acyclic for each $\left.F \in \mathcal{J}_{X_{s a}}\right\}$.

This category is generating, in fact if $\left\{G_{j}\right\}_{j}$ are R-constructible sheaves $\oplus_{j} \rho_{*} G_{j} \in \mathcal{P}_{X_{s a}}$ by Theorem 1.5.4. Moreover $\mathcal{P}_{X_{s a}}$ is stable by $\cdot \otimes K$, where $K \in \operatorname{Mod}_{\mathbb{R}-c}\left(k_{X}\right)$. In fact if $G \in \mathcal{P}_{X_{s a}}$ and $F \in \mathcal{J}_{X_{s a}}$ we have

$$
\operatorname{Hom}_{k_{X_{s a}}}(G \otimes K, F) \simeq \operatorname{Hom}_{k_{X_{s a}}}(G, \mathcal{H o m}(K, F))
$$

and $\operatorname{Hom}(K, F) \in \mathcal{J}_{X_{s a}}$ by Proposition 1.5.8.

THEOREM 1.5.16. The category $\left(\mathcal{P}_{X_{s a}}^{o p}, \mathcal{J}_{X_{s a}}\right)$ is injective with respect to the functor $\operatorname{Hom}_{k_{X s}}(\cdot, \cdot)$.

Proof. (i) Let $G \in \mathcal{P}_{X_{s a}}$ and consider an exact sequence $0 \rightarrow F^{\prime} \rightarrow$ $\rightarrow F \rightarrow F^{\prime \prime} \rightarrow 0$ with $F^{\prime} \in \mathcal{J}_{X_{s a}}$. We have to prove that the sequence

$$
0 \rightarrow \operatorname{Hom}_{k_{X_{s a}}}\left(G, F^{\prime}\right) \rightarrow \operatorname{Hom}_{k_{X_{s a}}}(G, F) \rightarrow \operatorname{Hom}_{k_{X_{s a}}}\left(G, F^{\prime \prime}\right) \rightarrow 0
$$

is exact. Since the functor $\operatorname{Hom}_{k_{X_{s a}}}(G, \cdot)$ is acyclic on quasi-injective sheaves we obtain the result.

(ii) Let $F \in \mathcal{J}_{X_{s a}}$, and let $0 \rightarrow G^{\prime} \rightarrow G \rightarrow G^{\prime \prime} \rightarrow 0$ be an exact sequence on $\mathcal{P}_{X_{s a}}$. Since the objects of $\mathcal{P}_{X_{s a}}$ are $\operatorname{Hom}_{k_{X_{s a}}}(\cdot, F)$-acyclic the sequence

$$
0 \rightarrow \operatorname{Hom}_{k_{X_{s u}}}\left(G^{\prime \prime}, F\right) \rightarrow \operatorname{Hom}_{k_{X_{s a}}}(G, F) \rightarrow \operatorname{Hom}_{k_{X_{s a}}}\left(G^{\prime}, F\right) \rightarrow 0
$$

is exact.

COROLlary 1.5.17. The category $\left(\mathcal{P}_{X_{s a}}^{o p}, \mathcal{J}_{X_{s a}}\right)$ is injective with respect to the functor $\mathcal{H}$ om $(\cdot, \cdot)$.

Proof. Let $G \in \mathcal{P}_{X_{s a}}$, and let $0 \rightarrow F^{\prime} \rightarrow F \rightarrow F^{\prime \prime} \rightarrow 0$ be an exact sequence with $F^{\prime} \in \mathcal{J}_{X_{s a}}$. We shall show that for each $U \in \mathrm{Op}\left(X_{s a}\right)$ the sequence

$$
0 \rightarrow \Gamma\left(U ; \mathcal{H o m}\left(G, F^{\prime}\right)\right) \rightarrow \Gamma(U ; \mathcal{H o m}(G, F)) \rightarrow \Gamma\left(U ; \mathcal{H o m}\left(G, F^{\prime \prime}\right)\right) \rightarrow 0
$$

is exact. This is equivalent to show that for each $U \in \mathrm{Op}\left(X_{s a}\right)$ the sequence

$$
0 \rightarrow \operatorname{Hom}_{k_{X_{s a}}}\left(G_{U}, F^{\prime}\right) \rightarrow \operatorname{Hom}_{k_{X_{s a}}}\left(G_{U}, F\right) \rightarrow \operatorname{Hom}_{k_{X_{s a}}}\left(G_{U}, F^{\prime \prime}\right) \rightarrow 0
$$

is exact. This follows since $G_{U} \in \mathcal{P}_{X_{s a}}$. The proof of the exactness in $\mathcal{P}_{X_{s a}}^{o p}$ is similar. 


\section{6 - The functor $\rho_{!}$.}

We have seen in $\S 1.1$ that the functor $\rho^{-1}: \operatorname{Mod}\left(k_{X_{s a}}\right) \rightarrow \operatorname{Mod}\left(k_{X}\right)$ has a left adjoint $\rho_{!}: \operatorname{Mod}\left(k_{X}\right) \rightarrow \operatorname{Mod}\left(k_{X_{s a}}\right)$. The functor $\rho_{!}$is fully faithful and exact. In particular, for $U \in \mathrm{Op}(X)$ one has $\rho_{!} k_{U} \simeq \underset{V \subset \subset U}{\lim } \rho_{*} k_{V}$, where $V \in \mathrm{Op}\left(X_{s a}\right)$.

Proposition 1.6.1. Let $S$ be a closed subset of $X$. Then $\rho_{!} k_{S} \simeq \underset{W \supset S}{\underset{\longrightarrow}{\lim }} \rho_{*} k_{\bar{W}}$, where $W \in \mathrm{Op}\left(X_{s a}\right)$.

Proof. (i) Let $U=X \backslash S$. Since $\rho_{\text {! }}$ is exact we have an exact sequence

$$
0 \rightarrow \rho_{!} k_{U} \rightarrow \rho_{!} k_{X} \rightarrow \rho_{!} k_{S} \rightarrow 0 .
$$

On the other hand, let $V \in \mathrm{Op}^{c}\left(X_{s a}\right)$ and $V \subset \subset U$. We have an exact sequence $0 \rightarrow k_{V} \rightarrow k_{X} \rightarrow k_{X \backslash V} \rightarrow 0$. Since $\rho_{*}$ is exact on $\operatorname{Mod}_{\mathbb{R}-c}\left(k_{X}\right)$ the sequence $0 \rightarrow \rho_{*} k_{V} \rightarrow \rho_{*} k_{X} \rightarrow \rho_{*} k_{X \backslash V} \rightarrow 0$ is exact. Applying the exact $\underset{V \subset \subset U}{\lim }$
we obtain an exact sequence

$$
0 \rightarrow \underset{V \subset \subset U}{\lim _{\longrightarrow}} \rho_{*} k_{V} \rightarrow \rho_{*} k_{X} \rightarrow \underset{V \subset \subset U}{\lim _{*}} \rho_{*} k_{X \backslash V} \rightarrow 0 .
$$

We have $\underset{V \subset \subset U}{\lim _{V}} \rho_{*} k_{V} \simeq \rho_{!} k_{U}$ and $\rho_{*} k_{X} \simeq \rho_{!} k_{X}$. Hence $\rho_{!} k_{S} \simeq \underset{V \subset \subset U}{\lim } \rho_{*} k_{X \backslash V}$.

(ii) We shall show that for each $U^{\prime} \in \mathrm{Op}^{c}\left(X_{s a}\right)$ the natural morphism

$$
\underset{V \subset \subset U}{\lim } \Gamma\left(U^{\prime} ; k_{X \backslash V}\right) \rightarrow \underset{W \supset S}{\lim _{\longrightarrow}} \Gamma\left(U^{\prime} ; k_{\bar{W}}\right)
$$

is an isomorphism. We shall see that for each $W \in \mathrm{Op}\left(X_{s a}\right)$ with $W \supset S$ there exists $W^{\prime} \in \mathrm{Op}\left(X_{s a}\right)$ such that $X \backslash \overline{W^{\prime}} \subset \subset U$ and $\bar{W} \cap U^{\prime}=\overline{W^{\prime}} \cap U^{\prime}$. Set $W^{\prime}=W \cup\left(X \backslash \overline{U^{\prime}}\right)$. Since $U^{\prime}$ is relatively compact, $X \backslash \overline{W^{\prime}} \subset \subset U$, and $\bar{W} \cap U^{\prime}=\overline{W^{\prime}} \cap U^{\prime}$ by construction. Then

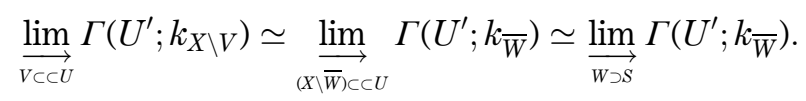

Notations 1.6.2. Let $Z=U \cap S$, where $U \in \mathrm{Op}(X)$ and let $S$ be a closed subset of $X$. Let $F \in \operatorname{Mod}\left(k_{X_{s a}}\right)$. We set for short ${ }_{Z} F=F \otimes \rho_{!} k_{Z}$

Lemma 1.6.3. Let $F \in \operatorname{Mod}\left(k_{X_{s a}}\right)$. Let $U \in \mathrm{Op}(X)$ and let $S$ be a closed subset of $X$. 


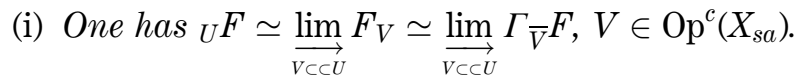

(ii) One has ${ }_{S} F \simeq \underset{W \supset S}{\lim _{W}} F_{\bar{W}} \simeq \underset{W \supset S}{\lim _{\longrightarrow}} \Gamma_{W} F, W \in \mathrm{Op}\left(X_{s a}\right)$.

Proof. (i) The first isomorphism is obvious. Let us show the second isomorphism. We have the chain of isomorphisms

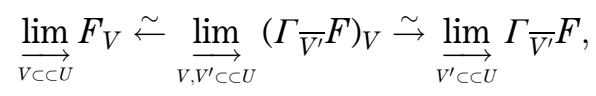

where $V, V^{\prime}$ range through the family of subanalytic open subsets of $X$.

The proof of (ii) is similar.

Proposition 1.6.4. Let $Z$ be a locally closed subset of $X$. Let $G \in \operatorname{Mod}_{\mathrm{R}-\mathrm{c}}\left(k_{X}\right)$ and $F \in \operatorname{Mod}\left(k_{X_{s a}}\right)$. Then ${ }_{Z} \mathcal{H o m}(G, F) \simeq \mathcal{H o m}\left(G,{ }_{Z} F\right)$.

Proof. (i) Let $U \in \mathrm{Op}(X)$. We have the chain of isomorphisms

$$
\begin{aligned}
{ }_{U} \mathcal{H o m}(G, F) & \simeq \underset{V \subset c U}{\lim } \Gamma_{\bar{V}} \mathcal{H o m}(G, F) \\
& \simeq \underset{V \subset \subset}{\lim } \mathcal{H o m}\left(G, \Gamma_{\bar{V}} F\right) \\
& \simeq \mathcal{H o m}\left(G, \underset{V \subset c U}{\lim } \Gamma_{\bar{V}} F\right) \\
& \simeq \mathcal{H o m}\left(G,{ }_{U} F\right),
\end{aligned}
$$

where $V \in \mathrm{Op}\left(X_{s a}\right)$. The third isomorphism follows from Proposition 1.3.4.

(ii) If $S$ is a closed subset of $X$ the proof is similar.

Proposition 1.6.5. Let $F \in \operatorname{Mod}\left(k_{X_{s a}}\right)$ be quasi-injective. Then $\rho_{!} K \otimes F$ is quasi-injective for each $K \in \operatorname{Mod}\left(k_{X}\right)$.

Proof. (i) Let us show the result when $K=k_{Z}$, for a locally closed subset $Z$ of $X$. Let $G \in \operatorname{Mod}_{\mathrm{R}-\mathrm{c}}^{c}\left(k_{X}\right)$. We have

$$
\begin{aligned}
\operatorname{Hom}_{k_{X_{s a}}}\left(G,{ }_{Z} F\right) & \simeq \Gamma\left(X ; \mathcal{H o m}\left(G,{ }_{Z} F\right)\right) \\
& \simeq \Gamma\left(X ;{ }_{Z} \mathcal{H o m}(G, F)\right) \\
& \simeq \Gamma\left(X ; \rho^{-1}{ }_{Z} \mathcal{H o m}(G, F)\right) \\
& \simeq \Gamma\left(X ;\left(\rho^{-1} \mathcal{H o m}(G, F)\right)_{Z}\right) .
\end{aligned}
$$

Since $F$ is quasi-injective, $\operatorname{Hom}(G, F)$ is quasi-injective. Then by Pro- 
position 1.5.15 the sheaf $\left(\rho^{-1} \mathcal{H o m}(G, F)\right)_{Z}$ is c-soft and it is injective with respect to the functor $\Gamma(X, \cdot)$. Hence the functor $\Gamma\left(X ; \rho^{-1} \mathcal{H o m}(\cdot, F)_{Z}\right)$ is exact on $\operatorname{Mod}_{\mathrm{R}-\mathrm{c}}^{\mathrm{c}}\left(k_{X}\right)$.

(ii) Let $K \in \operatorname{Mod}\left(k_{X}\right)$. There exists an epimorphism $\oplus_{i \in I} k_{U_{i}} \rightarrow K$ with $U_{i} \in \mathrm{Op}\left(X_{s a}\right)$ for each $i$. Let $K_{J}$ be the image of $\oplus_{i \in J} k_{U_{i}}$, with $J \subset I$ finite. We have $K \simeq \underset{J}{\lim } K_{J}$, hence $\rho_{!} K \simeq \underset{J}{\lim } \rho_{!} K_{J}$ since $\rho_{!}$commutes with $\underset{\longrightarrow}{\longrightarrow}$. It is enough to prove the result for $K_{J}$. We argue by induction on the cardinal of $J$. Set $K=K_{J}$. If $|J|=1$ then $K \simeq k_{Z}$ with $Z$ locally closed subset of $X$ and the result follows from (i).

Let us show $n-1 \Rightarrow n$. There is an epimorphism $\oplus_{i=1}^{n} k_{U_{i}} \rightarrow K$. Let $K_{1}$ be the image of $k_{U_{1}} \rightarrow K$ and let $K_{2}=K / K_{1}$. We have a commutative diagram

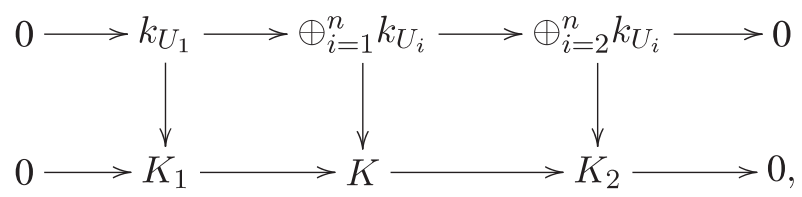

where the vertical arrows are surjective, and the rows are exact. By the exactness of $\rho_{!}$and $\otimes$ we obtain the exact sequence $0 \rightarrow \rho_{!} K_{1} \otimes F \rightarrow$ $\rightarrow \rho_{!} K \otimes F \rightarrow \rho_{!} K_{2} \otimes F \rightarrow 0$. By the inductive hypothesis $\rho_{!} K_{1} \otimes F$ and $\rho_{!} K_{2} \otimes F$ are quasi-injective, then $\rho_{!} K \otimes F$ is quasi-injective.

Proposition 1.6.6. Let $F \in \operatorname{Mod}\left(k_{X_{s a}}\right), G \in \operatorname{Mod}_{\mathrm{R}-\mathrm{c}}\left(k_{X}\right)$ and let $K \in$ $\operatorname{Mod}\left(k_{X}\right)$. One has the isomorphism $\mathcal{H o m}(G, F) \otimes \rho_{!} K \simeq \mathcal{H}$ om $\left(G, F \otimes \rho_{!} K\right)$.

Proof. Both sides are left exact with respect to $F$. Hence we may assume that $F$ is quasi-injective. Since quasi-injective sheaves are $\mathcal{H o m}(G, \cdot)$-injective, both sides are exact with respect to $K$. Moreover as a consequence of Proposition 1.3.4 both sides commute with filtrant lim with respect to $K$. We may reduce to the case $K=k_{U}$, with $U \in \mathrm{Op}\left(X_{s a}\right)$. Then the result follows from Proposition 1.6.4.

\section{Derived category.}

As usual, we denote $D\left(k_{X_{s a}}\right)$ the derived category of $\operatorname{Mod}\left(k_{X_{s a}}\right)$ and its full subcategory consisting of bounded (resp. bounded below, resp. bounded above) complexes is denoted by $D^{b}\left(k_{X_{s a}}\right)$ (resp. $D^{+}\left(k_{X_{s a}}\right)$, resp. $\left.D^{-}\left(k_{X_{s a}}\right)\right)$. 
2.1 - The category $D_{\mathbb{R}-c}^{b}\left(k_{X_{s a}}\right)$.

As usual we denote by $D_{\mathbb{R}-c}^{b}\left(k_{X}\right)$ (resp. $\left.D_{\mathbb{R}-c}^{b}\left(k_{X_{s a}}\right)\right)$ the full subcategory of $D^{b}\left(k_{X}\right)$ (resp. $D^{b}\left(k_{X_{s a}}\right)$ ) consisting of objects with $\mathbb{R}$-constructible cohomology.

Recall that $\rho: X \rightarrow X_{s a}$ is the natural morphism of sites. It induces the functor $\rho_{*}: \operatorname{Mod}\left(k_{X}\right) \rightarrow \operatorname{Mod}\left(k_{X_{s a}}\right)$.

Lemma 2.1.1. Let $F \in \operatorname{Mod}_{\mathbb{R}-\mathrm{c}}\left(k_{X}\right)$. Then $R^{j} \rho_{*} F=0$ for each $j \neq 0$.

Proof. The sheaf $R^{j} \rho_{*} F$ is the sheaf associated to the presheaf $V \rightarrow R^{j} \Gamma(V ; F)$. We have to show that $R^{j} \Gamma(V ; F)=0$ for $j \neq 0$ on a family of generators of the topology of $X_{s a}$. This means that for each $V \in \mathrm{Op}^{c}\left(X_{s a}\right)$ and for each $j \neq 0$, there exists $I$ finite and $\left\{V_{i}\right\}_{i \in I} \in \operatorname{Cov}\left(V_{s a}\right)$ such that $R^{j} \Gamma\left(V_{i} ; R \rho_{*} F\right) \simeq R^{j} \Gamma\left(V_{i} ; F\right)=0$.

We use the notation of [5]. There exists a locally finite stratification $\left\{X_{i}\right\}_{i \in I}$ of $X$ consisting of subanalytic subsets such that for all $j \in Z$ and all $i \in I$ the sheaf $\left.F\right|_{X_{i}}$ is locally constant. By the triangulation theorem there exist a simplicial complex $(S, \Delta)$ and a subanalytic homeomorphism $\psi:|S| \stackrel{\sim}{\rightarrow} X$ compatible with the stratification and such that $V$ is a finite union of the images by $\psi$ of open subsets $V(\sigma)$ of $|S|$, where $V(\sigma)=\bigcup_{\tau \in \Delta, \tau \supset \sigma}|\tau|$. By Proposition 8.1.4 of [5] we have $R^{j} \Gamma(\psi(V(\sigma)) ; F)=0$ for each $\sigma$ and for each $j \neq 0$. The result follows because $V=\bigcup_{\psi(|\sigma|) \subset V} \psi(V(\sigma))$.

Since $\mathbb{R}$-constructible sheaves are injective with respect to the functor $\rho_{*}$, the following diagram of derived categories is quasi-commutative.

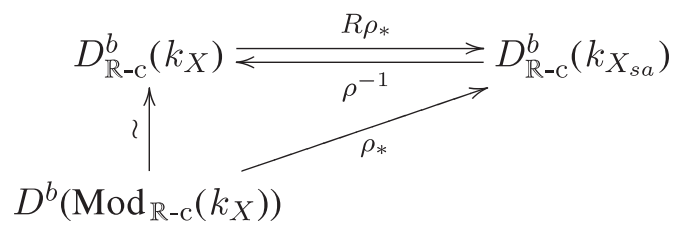

THEOREM 2.1.2. One has the equivalence of categories

$$
D_{\mathrm{R}-\mathrm{c}}^{b}\left(k_{X}\right) \simeq D^{b}\left(\operatorname{Mod}_{\mathbb{R}-\mathrm{c}}\left(k_{X}\right)\right) \simeq D_{\mathbb{R}-\mathrm{c}}^{b}\left(k_{X_{s a}}\right) .
$$


Proof. By dévissage, to prove the equivalence between $D^{b}\left(\operatorname{Mod}_{\mathbb{R}-c}\left(k_{X}\right)\right)$ and $D_{\mathrm{R}-\mathrm{c}}^{b}\left(k_{X_{s a}}\right)$ it is enough to check that the functor $\rho_{*}$ in (2.1) is fully faithful. We have $\rho^{-1} \circ \rho_{*} \simeq$ id and the result follows.

The equivalence between $D^{b}\left(\operatorname{Mod}_{\mathbb{R}-\mathrm{c}}\left(k_{X}\right)\right)$ and $D_{\mathbb{R}-\mathrm{c}}^{b}\left(k_{X}\right)$ was shown by Kashiwara in [4].

2.2 - Operations in the derived category.

Let us study the operations in the derived category of $\operatorname{Mod}\left(k_{X_{s a}}\right)$. Let $f: X \rightarrow Y$ be an analytic map. We will use quasi-injective objects and the results of $\S 1.5$ to derive the formulas of Section 1 .

Since $\operatorname{Mod}\left(k_{X_{s a}}\right)$ has enough injectives, then the derived functors

$$
\begin{aligned}
& R \mathcal{H o m}: D^{-}\left(k_{X_{s a}}\right)^{o p} \times D^{+}\left(k_{X_{s a}}\right) \rightarrow D^{+}\left(k_{X_{s a}}\right), \\
& R f_{*}: D^{+}\left(k_{X_{s a}}\right) \rightarrow D^{+}\left(k_{Y_{s a}}\right), \\
& R f_{! !}: D^{+}\left(k_{X_{s a}}\right) \rightarrow D^{+}\left(k_{Y_{s a}}\right),
\end{aligned}
$$

are well defined.

Proposition 2.2.1. Let $f: X \rightarrow Y$ be an analytic map. Then

(i) The functors $R f_{*}$ and $R$ Hom commute with $R \rho_{*}$.

(ii) The functors $R f_{*}$ and $R f_{! !}$commute with $\rho^{-1}$.

(iii) We have $R(g \circ f)_{*} \simeq R g_{*} \circ R f_{*}$ and $R(g \circ f)_{! !} \simeq R g_{! !} \circ R f_{! !}$.

(iv) The functor $R^{k} f_{! !}: \operatorname{Mod}\left(k_{X_{s a}}\right) \rightarrow \operatorname{Mod}\left(k_{Y_{s a}}\right)$ commutes with filtrant inductive limits for each $k \in Z$.

(v) If $F \in D^{+}\left(k_{X_{s a}}\right)$ and $f$ is proper on $\operatorname{supp}(F)$, then $R f_{! !} \simeq R f_{*}$.

Proof. (i) The functor $\rho_{*}$ sends injective sheaves to injective sheaves, then $R f_{*}$ and $R \mathcal{H}$ om commute with $R \rho_{*}$.

(ii) Since $\rho^{-1}$ has an exact left adjoint it sends injective sheaves to injective sheaves. Then $R f_{*}$ and $R f_{! !}$commute with $\rho^{-1}$.

(iii) The functor $f_{*}$ (resp. $f_{! !}$) sends injective sheaves to injective (resp. quasi-injective) sheaves. Then $R(g \circ f)_{*} \simeq R g_{*} \circ R f_{*}$ and $R(g \circ f)_{! !} \simeq$ $\simeq R g_{! !} \circ R f_{! !}$.

(iv) Quasi-injective objects of $\operatorname{Mod}\left(k_{X_{s a}}\right)$ are stable by filtrant lim, and the functor $f_{! !}$commutes with such limits. Then $R^{k} f_{! !}$commutes with filtrant $\stackrel{\lim }{\longrightarrow}$ for each $k \in Z$. 
(v) We can find a representative $F^{\prime}$ of $F$ in $K^{+}\left(\mathcal{J}_{X_{s a}}\right)$ with $f$ proper on $\operatorname{supp}\left(F^{\prime}\right)$. Then the result follows from the non derived case.

Proposition 2.2.2. Let $F=\underset{i}{\lim } F_{i}$ with $F_{i} \in \operatorname{Mod}\left(k_{X_{s a}}\right)$ and let $G \in D_{\mathbb{R}-\mathrm{c}}^{b}\left(k_{X}\right)$. One has $R^{k} \mathcal{H o m}(G, F) \simeq \underset{i}{\lim } R^{k} \mathcal{H o m}\left(G, F_{i}\right)$ for each $k \in Z_{\text {. }}$

Proof. There exists (see [8], Corollary 9.6.7) an inductive system of injective resolutions $I_{i}^{\bullet}$ of $F_{i}$. Then $\underset{i}{\lim } I_{i}^{\bullet}$ is a complex of quasi-injective objects quasi-isomorphic to $F$. Each object of $\left(\operatorname{Mod}_{\mathbb{R}-c}\left(k_{X}\right)^{o p}, \mathcal{J}_{X_{s a}}\right)$ is $\mathcal{H o m}(\cdot, \cdot)$-acyclic. Proposition 1.3.4 implies the isomorphism

$$
\mathcal{H o m}\left(G, \underset{i}{\lim } I_{i}^{\bullet}\right) \simeq \underset{i}{\lim } \mathcal{H o m}\left(G, I_{i}^{\bullet}\right)
$$

and the result follows.

Proposition 2.2.3. Let $F \in D^{+}\left(k_{X_{s a}}\right), G \in D_{\mathbb{R}-\mathrm{c}}^{b}\left(k_{X}\right)$ and let $K \in D^{+}\left(k_{X}\right)$. One has the isomorphism $R \mathcal{H} \operatorname{om}(G, F) \otimes \rho_{!} K \simeq R \mathcal{H o m}\left(G, F \otimes \rho_{!} K\right)$.

Proof. $\quad$ Let $I^{\bullet}$ be a quasi-injective resolution of $F$. By Proposition 1.6.5 we have that $I^{\bullet} \otimes \rho_{!} K$ is a complex of quasi-injective objects. Each object of $\left(\operatorname{Mod}_{\mathbb{R}-c}\left(k_{X}\right)^{o p}, \mathcal{J}_{X_{s a}}\right)$ is $\mathcal{H o m}(\cdot, \cdot)$-acyclic. Hence we are reduced to prove the isomorphism $\mathcal{H o m}\left(G, I^{\bullet}\right) \otimes \rho_{!} K \simeq \mathcal{H} \operatorname{om}\left(G, I^{\bullet} \otimes \rho_{!} K\right)$. The result follows from Proposition 1.6.6.

Proposition 2.2.4. Let $U \in \mathrm{Op}^{c}\left(X_{s a}\right)$. Let $F \in \operatorname{Mod}\left(k_{X_{s a}}\right)$ be quasiinjective. Then $F_{U}$ is $\Gamma(V, \cdot)$-acyclic for each $V \in \mathrm{Op}\left(X_{s a}\right)$.

Proof. Since $F_{U}$ has compact support, we may suppose that $V$ is relatively compact. Let $S=X \backslash U$. Since $F$ is quasi-injective and filtrant lim are exact, the morphism $\Gamma(V ; F) \rightarrow \underset{W \supset S \cap V}{\lim } \Gamma(W ; F) \stackrel{\sim}{\rightarrow} \Gamma\left(V ; F_{S}\right)$ is surjective. Consider the exact sequence $0 \rightarrow F_{U} \rightarrow F \rightarrow F_{S} \rightarrow 0$. We get the exact sequence

$$
0 \rightarrow \Gamma\left(V ; F_{U}\right) \rightarrow \Gamma(V ; F) \rightarrow \Gamma\left(V ; F_{S}\right) \rightarrow 0 .
$$

By Proposition 1.5.14 $F$ and $F_{S}$ are quasi-injective, hence $\Gamma(V ; \cdot)$-acyclic. This implies that $F_{U}$ is $\Gamma(V ; \cdot)$-acyclic.

Corollary 2.2.5. Let $f: X \rightarrow Y$ be a real analytic map and 
let $U \in \mathrm{Op}^{c}\left(X_{s a}\right)$ Let $F \in \operatorname{Mod}\left(k_{X_{s a}}\right)$ be quasi-injective. Then $F_{U}$ is $f_{! !}$-acyclic.

Proof. Since $F_{U}$ has compact support, $R f_{! !} F_{U} \simeq R f_{*} F_{U}$. The result follows because $F_{U}$ is $\Gamma\left(f^{-1}(V) ; \cdot\right)$-acyclic for each $V \in \mathrm{Op}\left(Y_{s a}\right)$.

LEMma 2.2.6. Let $F$ be quasi-injective object of $\operatorname{Mod}\left(k_{X_{s a}}\right)$ and let $G \in \operatorname{Mod}_{\mathbb{R}-c}^{c}\left(k_{X}\right)$. Then $F \otimes \rho_{*} G$ is $f_{! !-a c y c l i c}$.

Proof. Let $G \in \operatorname{Mod}_{\mathbb{R}_{-c}}^{c}\left(k_{X}\right)$. Then $G$ has a resolution

$$
0 \rightarrow \oplus_{i_{1} \in I_{1}} k_{U_{i_{1}}} \rightarrow \ldots \rightarrow \oplus_{i_{n} \in I_{n}} k_{U_{i_{n}}} \stackrel{\varphi}{\rightarrow} G \rightarrow 0,
$$

where $I_{j}$ is finite and $U_{i_{j}} \in \mathrm{Op}^{c}\left(X_{s a}\right)$ for each $i_{j} \in I_{j}, j \in\{1, \ldots, n\}$. Let us argue by induction on the length $n$ of the resolution.

If $n=1$, then $G$ is isomorphic to a finite sum $\oplus_{i} k_{U_{i}}$, with $U_{i} \in \mathrm{Op}^{c}\left(X_{s a}\right)$, and the result follows from Corollary 2.2.5.

Let us show $n-1 \Rightarrow n$. The sequence $0 \rightarrow \operatorname{ker} \varphi \rightarrow \oplus_{i_{n} \in I_{n}} k_{U_{i_{n}}} \rightarrow G \rightarrow 0$ is exact. The sheaf $\operatorname{ker} \varphi$ belongs to $\operatorname{Mod}_{\mathrm{R}-\mathrm{c}}^{c}\left(k_{X}\right)$ and it has a resolution of length $n-1$. Applying $F \otimes \rho_{*}(\cdot)$ we get the exact sequence

$$
0 \rightarrow F \otimes \rho_{*} \operatorname{ker} \varphi \rightarrow \oplus_{i_{n} \in I_{n}} F_{U_{i_{n}}} \rightarrow F \otimes \rho_{*} G \rightarrow 0 .
$$

By the induction hypothesis $F \otimes \rho_{*} \operatorname{ker} \varphi$ is $f_{! !}$-acyclic. Moreover $\oplus_{i_{n} \in I_{n}} F_{U_{i_{n}}}$ is $f_{! !}$-acyclic, then $F \otimes \rho_{*} G$ is $f_{! !-a c y c l i c}$.

Proposition 2.2.7. Let $F$ be quasi-injective object of $\operatorname{Mod}\left(k_{X_{s a}}\right)$ and let $G \in \operatorname{Mod}\left(k_{X_{s a}}\right)$. Then $F \otimes G$ is $f_{! !}$-acyclic.

Proof. Let $G \simeq \underset{i}{\lim } \rho_{*} G_{i}$ with $G_{i} \in \operatorname{Mod}_{\mathbb{R}-\mathrm{c}}^{c}\left(k_{X}\right)$ for each $i$. Since the functors $\otimes$ and $R^{k} f_{! !}$commute with filtrant $\underline{\text { lim }}$ we have

$$
R^{k} f_{! !}\left(F \otimes \underset{i}{\underset{\lim }{\longrightarrow}} \rho_{*} G_{i}\right) \simeq \underset{i}{\lim } R^{k} f_{! !}\left(F \otimes \rho_{*} G_{i}\right)=0
$$

if $k \neq 0$ by Lemma 2.2.6.

Proposition 2.2.8. Let $F \in D^{+}\left(k_{X_{s a}}\right)$ and $G \in D^{+}\left(k_{Y_{s a}}\right)$. Then

$$
R f_{! !} F \otimes G \simeq R f_{! !}\left(F \otimes f^{-1} G\right) .
$$


Proof. First assume that $F \in \operatorname{Mod}\left(k_{X_{s a}}\right)$ is injective. By Proposition 2.2.7 $F \otimes f^{-1} G$ is $f_{! !}$-acyclic.

Now let $F \in D^{+}\left(k_{X_{s a}}\right)$ and $G \in D^{+}\left(k_{Y_{s a}}\right)$. Let $F^{\prime}$ be a complex of injective sheaves quasi-isomorphic to $F$. Then

$$
R f_{! !} F \otimes G \simeq f_{! !} F^{\prime} \otimes G \simeq f_{! !}\left(F^{\prime} \otimes f^{-1} G\right) \simeq R f_{! !}\left(F \otimes f^{-1} G\right),
$$

where the second isomorphism follows from Proposition 1.4.5.

Now let us consider a cartesian square

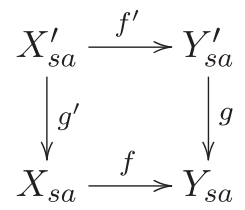

Proposition 2.2.9. Let $F \in D^{+}\left(k_{X_{s a}}\right)$. Then $g^{-1} R f_{! !} F \simeq R f_{! !}^{\prime} g^{\prime-1} F$.

Proof. We have an isomorphism $f_{! !}^{\prime} g^{\prime-1} \simeq g^{-1} f_{! !}$, and $R\left(g^{-1} f_{! !}\right) \simeq$ $\simeq g^{-1} R f_{! !}$since $g^{-1}$ is exact. Then we obtain a morphism $g^{-1} R f_{! !} \rightarrow R f_{! !}^{\prime} g^{\prime-1}$. It is enough to prove that for any $k \in Z$ and for any $F \in \operatorname{Mod}\left(k_{X_{s a}}\right)$ we have $g^{-1} R^{k} f_{! !} F \stackrel{\sim}{\rightarrow} R^{k} f_{! !}^{\prime} g^{\prime-1} F$. Since both sides commute with filtrant $\lim$, we may assume $F=\rho_{*} G$ with $G \in \operatorname{Mod}_{\mathbb{R}-c}^{c}\left(k_{X}\right)$. Moreover since $\operatorname{supp}(G)$ is compact, $f^{\prime}$ is proper on $\operatorname{supp}\left(g^{\prime-1} G\right)$. Then both sides commute with $\rho_{*}$ and the result follows from the corresponding one for classical sheaves.

As in classical sheaf theory, the Künneth formula follows from the projection formula and the base change formula.

Proposition 2.2.10. Consider a cartesian square

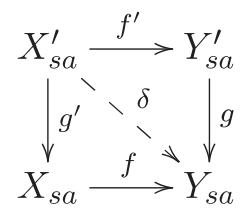

where $\delta=f g^{\prime}=g f^{\prime}$. There is a natural isomorphism

$$
R \delta_{! !}\left(g^{-1} F \otimes f^{\prime-1} G\right) \simeq R f_{! !} F \otimes R g_{! !} G
$$

for $F \in D^{+}\left(k_{X_{s a}}\right)$ and $G \in D^{+}\left(k_{Y_{s a}^{\prime}}\right)$. 
PRoof. Using the projection formula and the base change formula we deduce

$$
R f_{! !}^{\prime}\left(g^{\prime-1} F \otimes f^{\prime-1} G\right) \simeq R f_{! !}^{\prime} g^{\prime-1} F \otimes G \simeq g^{-1} R f_{! !} F \otimes G .
$$

Using the projection formula once again we find

$$
R g_{! !} R f_{! !}^{\prime}\left(g^{\prime-1} F \otimes f^{\prime-1} G\right) \simeq R g_{! !}\left(g^{-1} R f_{! !} F \otimes G\right) \simeq R f_{! !} F \otimes R g_{! !} G
$$

and the result follows since $R \delta_{! !} \simeq R g_{! !} \circ R f_{! !}^{\prime}$.

The following isomorphism is the analogue for subanalytic sheaves of Lemma 5.2 .8 of [7]

Proposition 2.2.11. Let $G \in D_{\mathrm{R}-\mathrm{c}}^{b}\left(k_{Y}\right)$ and let $F \in D^{+}\left(k_{X_{s a}}\right)$. Then the natural morphism

$$
R f_{! !} R \mathcal{H o m}\left(f^{-1} G, F\right) \rightarrow R \mathcal{H o m}\left(G, R f_{! !} F\right)
$$

is an isomorphism.

Proof. The morphism is obtained as in the non derived case. Let us show that it is an isomorphism. Let $F^{\prime}$ be a complex of injective sheaves quasi-isomorphic to $F$. Then

$$
\begin{aligned}
R f_{! !} \mathcal{H o m}\left(f^{-1} G, F\right) & \simeq f_{! !} \mathcal{H o m}\left(f^{-1} G, F^{\prime}\right) \\
& \simeq \mathcal{H o m}\left(G, f_{! !} F^{\prime}\right) \\
& \simeq R \mathcal{H o m}\left(G, R f_{! !}\right),
\end{aligned}
$$

where the second isomorphism follows from Proposition 1.4.7.

2.3 - Vanishing theorems on $\operatorname{Mod}\left(k_{X_{s a}}\right)$.

In $\S 2.3$ we prove some results on the vanishing of the cohomology of sheaves on a subanalytic site.

Definition 2.3.1. The quasi-injective dimension of the category $\operatorname{Mod}\left(k_{X_{s a}}\right)$ is the smallest $n \in \mathbb{N} \cup\{\infty\}$ such that for any $F \in \operatorname{Mod}\left(k_{X_{s a}}\right)$ there exists an exact sequence

$$
0 \rightarrow F \rightarrow \mathrm{I}^{0} \rightarrow \cdots \rightarrow I^{n} \rightarrow 0
$$

with $I^{j}$ quasi-injective for $0 \leq j \leq n$. 
Proposition 2.3.2. The category $\operatorname{Mod}\left(k_{X_{s a}}\right)$ has finite quasi-injective dimension.

Proof. Let $F \in \operatorname{Mod}\left(k_{X_{s a}}\right)$. Then $F=\underset{i}{\lim } \rho_{*} F_{i}$, with $F_{i} \in \operatorname{Mod}_{\mathrm{R}-\mathrm{c}}^{c}\left(k_{X}\right)$. There exists (see [8], Corollary 9.6.7) an inductive system of injective resolutions $I_{i}^{\bullet}$ of $F_{i}$. By Proposition 3.3.11 of [5], the category $\operatorname{Mod}\left(k_{X}\right)$ has finite homological dimension. Then we may assume that $I_{i}^{\bullet}$ has length $N_{0}<\infty$ for each $i$. Since $F_{i}$ is $\rho_{*}$-injective for each $i, \rho_{*} I_{i}^{\bullet}$ is an injective resolution of $\rho_{*} F_{i}$ of length $N_{0}$. Taking the inductive limit we find that $\underset{i}{\lim } \rho_{*} I_{i}^{\bullet}$ is a resolution of $F$ of length $N_{0}$, and $\underset{i}{\lim } \rho_{*} I_{i}^{j} \in \mathcal{J}_{X_{s a}}$ for each $j$.

Corollary 2.3.3. Let $f: X \rightarrow Y$ be a real analytic map, and let $F \in \operatorname{Mod}_{\mathbb{R}-\mathrm{c}}\left(k_{X}\right)$. The functors $f_{*}, f_{! !}$and $\mathcal{H o m}(F, \cdot)$ have finite cohomological dimension.

Proposition 2.3.4. Let $F \in \operatorname{Mod}\left(k_{X}\right)$ and let $G \in \operatorname{Mod}\left(k_{X_{s a}}\right)$. There exists a finite $j_{0} \in \mathbb{N}$ such that

$$
R^{j} \mathcal{H o m}\left(\rho_{!} F, G\right)=0 \text { for } j>j_{0} \text {. }
$$

Proof. Let $U \in \mathrm{Op}\left(X_{s a}\right)$. We have the chain of isomorphisms

$$
\begin{aligned}
R \Gamma\left(U ; R \mathcal{H} \operatorname{com}\left(\rho_{!} F, G\right)\right) & \simeq \operatorname{RHom}_{k_{x_{s}}}\left(\rho_{!} F, R \Gamma_{U} G\right) \\
& \simeq \operatorname{RHom}_{k_{X}}\left(F, \rho^{-1} R \Gamma_{U} G\right) .
\end{aligned}
$$

The functor $\Gamma_{U}$ has finite cohomological dimension, and the homological dimension of the category $\operatorname{Mod}\left(k_{X}\right)$ is finite. Hence we can find a finite $j_{0} \in \mathbb{N}$ such that $R^{j} \Gamma\left(U ; R \mathcal{H} \operatorname{Hom}\left(\rho_{!} F, G\right)\right)$ vanishes for $j>j_{0}$ and for each $U \in \mathrm{Op}\left(X_{s a}\right)$. This shows the result.

REMARK 2.3.5. We have seen that the functor $\mathcal{H o m}(F, \cdot)$ has finite cohomological dimension when $F$ is $\mathbb{R}$-constructible and when $F=\rho_{!} G$ with $G \in \operatorname{Mod}\left(k_{X}\right)$. We do not know if the cohomological dimension is finite for any $F \in \operatorname{Mod}\left(k_{X_{s a}}\right)$. Indeed we do not know if the homological dimension of $\operatorname{Mod}\left(k_{X_{s a}}\right)$ is finite or not. 


\section{4 - Duality.}

In the following we find a right adjoint to the functor $R f_{! !}$, denoted by $f^{!}$. The construction is not inspired to the classical one as in [7], but it is done via Brown representability (we refer to [8] for an exposition on this subject). We calculate $f^{!}$by decomposing $f$ as the composite of a closed embedding and a submersion.

The subcategory $\mathcal{J}_{X_{s a}}$ of quasi-injective objects and the functor $f_{! !}$have the following properties:

$$
\left\{\begin{array}{l}
\text { (i) } \mathcal{J}_{X_{s a}} \text { is cogenerating, } \\
\text { (ii) } \operatorname{Mod}\left(k_{X_{s a}}\right) \text { has finite quasi-injective dimension, } \\
\text { (iii) } \mathcal{J}_{X_{s a}} \text { is } f_{! !-\text {injective, }} \\
\text { (iv) } \mathcal{J}_{X_{s a}} \text { is closed by small } \oplus, \\
\text { (v) } f_{! !} \text {commutes with small } \oplus .
\end{array}\right.
$$

As a consequence of the Brown representability theorem (see [8], Corollary 14.3.7 for details) we find a right adjoint to the functor $R f_{! !}$. Remark that the functor $R f_{! !}$extends to a functor $R f_{! !}: D\left(k_{X_{s a}}\right) \rightarrow D\left(k_{Y_{s a}}\right)$.

THeOREM 2.4.1. The functor $R f_{! !}: D\left(k_{X_{s a}}\right) \rightarrow D\left(k_{Y_{s a}}\right)$ admits a right adjoint. We denote by $f^{!}: D\left(k_{Y_{s a}}\right) \rightarrow D\left(k_{X_{s a}}\right)$ the adjoint functor.

\section{Corollary 2.4.2. Let $G \in D^{+}\left(k_{Y_{s a}}\right)$. Then $f^{!} G \in D^{+}\left(k_{X_{s a}}\right)$.}

Proof. We may reduce to the case that $G \in D^{\geq 0}\left(k_{Y_{s a}}\right)$. Let $N_{0}$ be the quasi-injective dimension of $\operatorname{Mod}\left(k_{X_{s a}}\right)$ and let $F \in D^{\leq-N_{0}-1}\left(k_{X_{s a}}\right)$. Then $R f_{! !} F \in D^{\leq-1}\left(k_{Y_{s a}}\right)$ and

$$
\operatorname{Hom}_{D\left(k_{Y_{s a}}\right)}\left(R f_{! !} F, G\right) \simeq \operatorname{Hom}_{D\left(k_{X_{s a}}\right)}\left(F, f^{!} G\right)=0 .
$$

Hence for each $F \in D^{\leq-N_{0}-1}\left(k_{X_{s a}}\right)$ we have $\operatorname{Hom}_{D\left(k_{X_{s a}}\right)}\left(F, f^{!} G\right)=0$. Set for short $a=-N_{0}-1$, if $F=\tau^{\leq a} f^{!} G$ we have

$$
\operatorname{Hom}_{D\left(k_{X_{s} a}\right)}\left(\tau^{\leq a} f^{!} G, f^{!} G\right) \simeq \operatorname{Hom}_{D \leq a}\left(k_{X_{s a}}\right)\left(\tau^{\leq a} f^{!} G, \tau^{\leq a} f^{!} G\right)=0 .
$$

This implies $\tau^{\leq a} f^{!} G=0$, hence $f^{!} G \in D^{+}\left(k_{X_{s a}}\right)$.

REMARK 2.4.3. As in classical sheaf theory one can prove that for $F \in$ $\in D^{b}\left(k_{X_{s a}}\right)$ and $G \in D^{+}\left(k_{Y_{s a}}\right)$ one has $R f_{*} R \mathcal{H o m}\left(F, f^{!} G\right) \simeq R \mathcal{H o m}\left(R f_{! !} F, G\right)$. 
REMARK 2.4.4. As in classical sheaf theory, one can prove by adjunction the dual projection formula and the dual base change formula.

Proposition 2.4.5. The functor $f$ ! commutes with $R \rho_{*}$, and the functor $H^{k} f^{!}: \operatorname{Mod}\left(k_{Y_{s a}}\right) \rightarrow \operatorname{Mod}\left(k_{X_{s a}}\right)$ commutes with filtrant $\underline{\longrightarrow}$.

Proof. Since $R f_{! !}$commutes with $\rho^{-1}$, then $f^{!}$commutes with $R \rho_{*}$ by adjunction.

Let us show that $H^{k} f$ ! commutes with lim. Let $\left\{F_{i}\right\}_{i}$ be a filtrant inductive system in $\operatorname{Mod}\left(k_{Y_{s a}}\right)$. Remark that $\underset{i}{\stackrel{\lim }{\longrightarrow}} H^{k} f^{!} F_{i}\left(\right.$ resp. $\left.H^{k} f^{!} \underset{i}{\lim } F_{i}\right)$ is the sheaf associated to the presheaf $U \mapsto \underset{i}{\lim } R^{k} \Gamma\left(U ; f^{!} F_{i}\right) \quad$ (resp. $\left.U \mapsto R^{k} \Gamma\left(U ; f^{!} \underset{i}{\lim } F_{i}\right)\right)$, for $U \in \mathrm{Op}^{c}\left(X_{s a}\right)$.

We will show the isomorphism $R^{k} \Gamma\left(U ; f^{!} \underset{i}{\lim } F_{i}\right) \stackrel{\sim}{\underset{i}{\lim }} R^{k} \Gamma\left(U ; f^{!} F_{i}\right)$ for each $U \in \mathrm{Op}^{c}\left(X_{s a}\right)$. By adjunction it is enough to prove the isomorphism $R^{k} \operatorname{Hom}_{k_{Y_{s a}}}\left(R f_{!} k_{U}, \underset{i}{\lim } F_{i}\right) \simeq \underset{i}{\lim } R^{k} \operatorname{Hom}_{k_{Y_{s a}}}\left(R f_{!} k_{U}, F_{i}\right) \quad$ (remark that we have $R f_{! !} k_{U} \simeq R f_{!} k_{U} \simeq R f_{*} k_{U}$ since $\left.U \in \mathrm{Op}^{c}\left(X_{s a}\right)\right)$.

Let $\mathcal{J}_{Y_{s a}}$ be the family of quasi-injective objects of $\operatorname{Mod}\left(k_{Y_{s a}}\right)$. Each object of $\left(\operatorname{Mod}_{\mathbb{R}-c}^{c}\left(k_{Y}\right)^{o p}, \mathcal{J}_{Y_{s a}}\right)$ is $\operatorname{Hom}_{k_{Y_{s a}}}(\cdot, \cdot)$-acyclic. Moreover $\mathcal{J}_{Y_{s a}}$ is stable by filtrant inductive limits. There exists (see [8], Corollary 9.6.7) an inductive system of injective resolutions $I_{i}^{\bullet}$ of $F_{i}$. Then $\lim _{\longrightarrow} I_{i}^{\bullet}$ is a quasiinjective resolution of $\underset{i}{\lim } F_{i}$. We have

$$
\operatorname{Hom}_{K^{+}\left(k_{Y_{s a}}\right)}\left(R f_{!} k_{U}, \underset{i}{\lim } I_{i}^{\bullet}\right) \simeq \underset{i}{\lim } \operatorname{Hom}_{K^{+}\left(k_{Y_{s a}}\right)}\left(R f_{!} k_{U}, I_{i}^{\bullet}\right)
$$

and the result follows.

Corollary 2.4.6. Let $F \in D^{b}\left(k_{Y_{s a}}\right)$. Then $f^{!} F \in D^{b}\left(k_{X_{s a}}\right)$.

Proof. We may reduce to the case $F \in \operatorname{Mod}\left(k_{Y_{s a}}\right)$. Then $F \simeq \underset{i}{\lim } \rho_{*} F_{i}$ with $F_{i} \in \operatorname{Mod}_{\mathbb{R}-\mathrm{c}}\left(k_{Y}\right)$ for each $i$. By Proposition 2.4.5 we have

$$
H^{k} f^{!} F \simeq H^{k} f^{!} \underset{i}{\lim } \rho_{*} F_{i} \simeq \underset{i}{\lim } \rho_{*} H^{k} f^{!} F_{i},
$$

and $H^{k} f^{!} F_{i}=0$ if $k>j_{0}$ for a fixed $j_{0} \in \mathbb{N}$ and for each $i$. 
The following result is the analogue for subanalytic sheaves of Proposition 5.3 .9 of [7].

Proposition 2.4.7. Let $F \in D^{+}\left(k_{Y_{s a}}\right)$ and let $G \in D^{+}\left(k_{Y}\right)$. Then one has the isomorphism $f^{!}\left(F \otimes \rho_{!} G\right) \simeq f^{!} F \otimes \rho_{!} f^{-1} G$.

Proof. We have the chain of morphisms

$$
R f_{! !}\left(f^{!} F \otimes \rho_{!} f^{-1} G\right) \simeq R f_{! !} f^{!} F \otimes \rho_{!} G \rightarrow F \otimes \rho_{!} G,
$$

by adjunction we obtain the desired morphism. To prove that it is an isomorphism it is enough to show $R^{k} \Gamma\left(U ; f^{!}\left(F \otimes \rho_{!} G\right)\right) \simeq R^{k} \Gamma\left(U ; f^{!} F \otimes \rho_{!} f^{-1} G\right)$ for each $U \in \mathrm{Op}^{c}\left(X_{s a}\right)$ and each $k \in Z$. We have the chain of isomorphisms

$$
\begin{aligned}
R^{k} \Gamma\left(U ; f^{!}\left(F \otimes \rho_{!} G\right)\right) & \simeq R^{k} \operatorname{Hom}_{k_{Y_{s a}}}\left(R f_{! !} k_{U}, F \otimes \rho_{!} G\right) \\
& \simeq R^{k} \operatorname{Hom}_{k_{Y_{s a}}}\left(k_{Y}, R \mathcal{H o m}\left(R f_{! !} k_{U}, F \otimes \rho_{!} G\right)\right) \\
& \simeq R^{k} \operatorname{Hom}_{k_{Y_{s a}}}\left(k_{Y}, R \mathcal{H o m}\left(R f_{! !} k_{U}, F\right) \otimes \rho_{!} G\right) \\
& \simeq R^{k} \operatorname{Hom}_{k_{Y_{s a}}}\left(k_{Y}, R f_{! !} R \mathcal{H o m}\left(k_{U}, f^{!} F\right) \otimes \rho_{!} G\right) \\
& \simeq R^{k} \operatorname{Hom}_{k_{Y_{s a}}}\left(k_{Y}, R f_{! !}\left(R \mathcal{H o m}\left(k_{U}, f^{!} F\right) \otimes f^{-1} \rho_{!} G\right)\right) \\
& \simeq R^{k} \operatorname{Hom}_{k_{Y_{s a}}}\left(k_{Y}, R f_{! !}\left(R \mathcal{H o m}\left(k_{U}, f^{!} F \otimes \rho_{!} f^{-1} G\right)\right)\right) \\
& \simeq R^{k} \operatorname{Hom}_{k_{X_{s a}}}\left(k_{U}, f^{!} F \otimes \rho_{!} f^{-1} G\right) .
\end{aligned}
$$

Here the fourth and the last isomorphism follow from the fact that since $k_{U}$ has compact support, then $R \mathcal{H}$ om $\left(k_{U}, K\right)$ has compact support for any $K \in D^{+}\left(k_{X_{s a}}\right)$ and $R f_{! !} R \mathcal{H o m}\left(k_{U}, K\right) \simeq R f_{*} R \mathcal{H o m}\left(k_{U}, K\right)$.

Proposition 2.4.8. Let $F \in D^{+}\left(k_{Y_{s a}}\right)$, and let $f: X \rightarrow Y$ be a closed embedding. Then $f^{!} F \simeq f^{-1} R \mathcal{H} \operatorname{om}\left(k_{X}, F\right)$ and id $\stackrel{\sim}{\rightarrow} f^{!} R f_{! !}$.

PRoof. Since $f$ is proper, then $R f_{*} \simeq R f_{! !}$. We have the isomorphisms $R f_{*} f^{!} F \simeq R f_{*} R \mathcal{H o m}\left(k_{X}, f^{!} F\right) \simeq R \mathcal{H o m}\left(k_{X}, F\right)$. Since $f^{-1} R f_{*} f^{!} F \simeq f^{!} F$, then $f^{!} F \simeq f^{-1} R \mathcal{H o m}\left(k_{X}, F\right)$.

Let $F^{\prime} \in D^{+}\left(k_{X_{s a}}\right)$. We have the isomorphisms

$$
f^{!} R f_{*} F^{\prime} \simeq f^{-1} R \mathcal{H o m}\left(k_{X}, R f_{*} F^{\prime}\right) \simeq f^{-1} R f_{*} R \mathcal{H o m}\left(k_{X}, F^{\prime}\right) \simeq f^{-1} R f_{*} F^{\prime},
$$

and $f^{-1} R f_{*} F^{\prime} \simeq F^{\prime}$ since $f$ is a closed embedding. 
Recall that $f$ is a topological submersion (of fiber dimension $n$ ) if locally on $\mathrm{X}, f$ is isomorphic to the projection $Y \times \mathbb{R}^{n} \rightarrow Y$.

Proposition 2.4.9. Assume that $f$ is a topological submersion. Then for $F \in D^{+}\left(k_{Y_{s a}}\right)$ one has the isomorphism $f^{-1} F \otimes f^{!} k_{Y} \stackrel{\sim}{\rightarrow} f^{!} F$.

Proof. We have the chain of morphisms

$$
R f_{! !}\left(f^{-1} F \otimes f^{!} k_{Y}\right) \simeq F \otimes R f_{! !} f^{!} k_{Y} \rightarrow F \otimes k_{Y} \simeq F,
$$

by adjunction we obtain the desired morphism.

Let us show that it is an isomorphism. We may reduce to the case $F \simeq \underset{i}{\lim } \rho_{*} F_{i} \in \operatorname{Mod}\left(k_{Y_{s} a}\right)$. We have the chain of isomorphisms

$$
\begin{aligned}
H^{k}\left(f^{-1} \underset{i}{\lim _{*}} \rho_{*} F_{i} \otimes f^{!} \rho_{*} k_{Y}\right) & \simeq \underset{i}{\lim _{*}} \rho_{*} H^{k}\left(f^{-1} F_{i} \otimes f^{!} k_{Y}\right) \\
& \simeq \underset{i}{\lim } \rho_{*} H^{k} f^{!} F_{i} \\
& \simeq H^{k} f^{!} \underset{i}{\lim } \rho_{*} F_{i} .
\end{aligned}
$$

Using these results we can calculate explicitly the functor $f^{!}$. Let $f: X \rightarrow Y$ be an analytic map. We decompose it as the composite of a closed embedding and a submersion. In fact

$$
f: X \stackrel{j}{\hookrightarrow} X \times Y \stackrel{p}{\rightarrow} Y
$$

where $p$ is the projection and $j$ is the graph embedding $j(x)=(x, f(x))$. Let $F \in D^{+}\left(k_{Y_{s a}}\right)$. Applying Propositions 2.4.8 and 2.4.9 we get

$$
f^{!} F \simeq j^{-1} R \mathcal{H o m}\left(k_{j(X)}, p^{-1} F \otimes p^{!} k_{Y}\right) .
$$

CoRollary 2.4.10. Assume that $f$ is a topological submersion. Then:

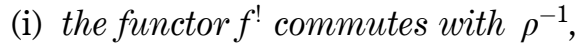

(ii) the functor $R f_{! !}$commutes with $\rho_{!}$.

Proof. (i) One has the chain of isomorphisms

$$
\begin{aligned}
\rho^{-1}\left(f^{-1} F \otimes f^{!} \rho_{*} k_{Y}\right) & \simeq \rho^{-1} f^{-1} F \otimes \rho^{-1} f^{!} \rho_{*} k_{Y} \\
& \simeq f^{-1} \rho^{-1} F \otimes \rho^{-1} f^{!} \rho_{*} k_{Y} \\
& \simeq f^{-1} \rho^{-1} F \otimes \rho^{-1} \rho_{*} f^{!} k_{Y} \\
& \simeq f^{-1} \rho^{-1} F \otimes f^{!} k_{Y} .
\end{aligned}
$$


The result follows from Proposition 2.4.9.

(ii) The result follows by adjunction.

Proposition 2.4.11. Assume that $f$ is a topological submersion and moreover that $R f_{!} f^{!} k_{Y} \simeq k_{Y}$. Then for $F \in D^{+}\left(k_{Y_{s a}}\right)$ the morphism $R f_{*} f^{-1} F \rightarrow F$ is an isomorphism.

Proof. First let us show that $R f_{! !} f^{!} k_{Y} \simeq k_{Y}$. We have the chain of isomorphisms

$$
\begin{aligned}
R f_{! !} f^{!} \rho_{*} k_{Y} & \simeq R f_{! !} \rho_{*} f^{!} k_{Y} \\
& \simeq R f_{! !} \rho_{!} f^{!} k_{Y} \\
& \simeq \rho_{!} R f_{!} f^{!} k_{Y} \\
& \simeq \rho_{!} k_{Y} \\
& \simeq \rho_{*} k_{Y},
\end{aligned}
$$

where the second isomorphism follows because $f^{!} k_{Y}$ is locally constant and the third from Corollary 2.4.10 (ii). It follows from Proposition 2.4.9 that $f^{-1} F \simeq R \mathcal{H o m}\left(f^{!} k_{Y}, f^{!} F\right)$. Then we have the chain of isomorphisms

$$
\begin{aligned}
R f_{*} f^{-1} F & \simeq R f_{*} R \mathcal{H o m}\left(f^{!} k_{Y}, f^{!} F\right) \\
& \simeq R \mathcal{H o m}\left(R f_{! !} f^{!} k_{Y}, F\right) \\
& \simeq F
\end{aligned}
$$

\section{Examples of applications.}

In this Section we give some example of subanalytic sheaves. Let $X$ be a real analytic manifold, and let $X_{s a}$ be the associated subanalytic site. We first introduce sheaves of $\mathcal{R}$-modules, where $\mathcal{R}$ is a sheaf of $k$ algebras on $X_{s a}$. Let $\mathcal{D}_{X}$ be the sheaf of finite order differential operators on $X$. We define the $\rho_{!} \mathcal{D}_{X}$-modules $\mathcal{O}_{X}^{t}$ and $\mathcal{O}_{X}^{\mathrm{W}}$ of tempered and Whitney holomorphic functions respectively. References are made to [8] for an exposition on sheaves of rings on a Grothendieck topology. 
3.1 - Modules over a $k_{X_{s a}}$-algebra.

A sheaf of $k_{X_{s a}}$-algebras (or a $k_{X_{s u}}$-algebra, for short) is an object $\mathcal{R} \in \operatorname{Mod}\left(k_{X_{s a}}\right)$ such that $\Gamma(U ; \mathcal{R})$ is a $k$-algebra for each $U \in \mathrm{Op}\left(X_{s a}\right)$ and the restriction maps are morphisms of algebras. Let us denote by $\mathcal{R}^{o p}$ the opposite $k_{X_{s a}}$-algebra.

Let $\mathcal{R}$ be a $k_{X_{s a}}$-algebra and denote by $\operatorname{Mod}(\mathcal{R})$ the category of sheaves of (left) $\mathcal{R}$-modules. The category $\operatorname{Mod}(\mathcal{R})$ is a Grothendieck category and the family $\left\{\mathcal{R}_{U}\right\}_{U \in \mathrm{Op}{ }^{c}\left(X_{s a}\right)}$ is a small system of generators. Moreover the forgetful functor for $: \operatorname{Mod}(\mathcal{R}) \rightarrow \operatorname{Mod}\left(k_{X_{s a}}\right)$ is exact.

In this Section we shall extend some results on $k_{X_{s a}}$-modules, by replacing $k_{X_{s a}}$ with $\mathcal{R}$. Since the formalism is similar to that we developed previously we shall not give proofs. The functors

$$
\begin{array}{r}
\mathcal{H o m}_{\mathcal{R}}: \operatorname{Mod}(\mathcal{R})^{o p} \times \operatorname{Mod}(\mathcal{R}) \rightarrow \operatorname{Mod}\left(k_{X_{s a}}\right), \\
\otimes_{\mathcal{R}}: \operatorname{Mod}\left(\mathcal{R}^{o p}\right) \times \operatorname{Mod}(\mathcal{R}) \rightarrow \operatorname{Mod}\left(k_{X_{s a}}\right)
\end{array}
$$

are well defined. Let us summarize their properties:

- the functor $\mathcal{H o m}_{\mathcal{R}}$ is left exact,

- the functor $\otimes_{\mathcal{R}}$ is right exact and commutes with $\underline{\mathrm{lim}}$.

Let $X, Y$ be two real analytic manifolds, and let $f: X \rightarrow Y$ be a morphism of real analytic manifolds. Let $\mathcal{R}$ be a $k_{Y_{s a}}$-algebra. The functors $f^{-1}, f_{*}$ and $f_{! !}$induce functors

$$
\begin{gathered}
f^{-1}: \operatorname{Mod}(\mathcal{R}) \rightarrow \operatorname{Mod}\left(f^{-1} \mathcal{R}\right), \\
f_{*}: \operatorname{Mod}\left(f^{-1} \mathcal{R}\right) \rightarrow \operatorname{Mod}(\mathcal{R}), \\
f_{!}: \operatorname{Mod}\left(f^{-1} \mathcal{R}\right) \rightarrow \operatorname{Mod}(\mathcal{R}) .
\end{gathered}
$$

Let us summarize their properties:

- the functor $f^{-1}$ is exact and commutes with $\underline{\mathrm{lim}}$ and $\otimes_{\mathcal{R}}$,

- the functor $f_{*}$ is left exact and commutes with $\underset{\longleftarrow}{\mathrm{lim}}$,

- $\left(f^{-1}, f_{*}\right)$ is a pair of adjoint functors,

- the functor $f_{! !}$is left exact and commutes with filtrant $\stackrel{\text { lim. }}{\longrightarrow}$.

Now we consider the derived category of sheaves of $\mathcal{R}$-modules.

DeFinition 3.1.1. An object $F \in \operatorname{Mod}(\mathcal{R})$ is flat if the functor $\operatorname{Mod}\left(\mathcal{R}^{o p}\right) \ni G \rightarrow G \otimes_{\mathcal{R}} F$ is exact. 
Small direct sums and filtrant inductive limits of flat $\mathcal{R}$-modules are flat. Since the generators of $\operatorname{Mod}(\mathcal{R})$ are flat, then the subcategory of $\operatorname{Mod}(\mathcal{R})$ consisting of flat modules is generating. Thanks to flat objects we can find a left derived functor $\otimes_{\mathcal{R}}^{L}$ of the tensor product $\otimes_{\mathcal{R}}$.

DeFINITION 3.1.2. An object $F \in \operatorname{Mod}(\mathcal{R})$ is quasi-injective if its image via the forgetful functor for $: \operatorname{Mod}(\mathcal{R}) \rightarrow \operatorname{Mod}\left(k_{X_{s a}}\right)$ is quasi-injective.

Let $X, Y$ be two real analytic manifolds, and let $f: X \rightarrow Y$ be a real analytic map. Let $\mathcal{R}$ be a $k_{Y_{s a}}$-algebra. As in $\S 1.5$ one can prove that quasiinjective objects are injective with respect to the functors $f_{*}$ and $f_{! !}$. The functors $R f_{*}$ and $R f_{! !}$are well defined and projection formula, base change formula and Künneth formula remain valid for $\mathcal{R}$-modules. Moreover hypothesis (2.2) are satisfied and we have

THeOREM 3.1.3. The functor $R f_{! !}: D^{+}\left(f^{-1} \mathcal{R}\right) \rightarrow D^{+}(\mathcal{R})$ admits a right adjoint. We denote by $f^{!}: D^{+}(\mathcal{R}) \rightarrow D^{+}\left(f^{-1} \mathcal{R}\right)$ the adjoint functor.

\section{2 - Sheaves of $\rho_{!} \mathcal{R}$-modules.}

We will consider the case where the ring is $\rho_{!} \mathcal{R}$, where $\mathcal{R}$ is a sheaf of $k_{X}$-algebras. We will also assume the following hypothesis:

$\mathcal{R}$ has finite flat dimension.

The functor $\rho_{!}$induces an exact functor $\operatorname{Mod}(\mathcal{R}) \rightarrow \operatorname{Mod}\left(\rho_{!} \mathcal{R}\right)$ which is left adjoint to $\rho^{-1}: \operatorname{Mod}\left(\rho_{!} \mathcal{R}\right) \rightarrow \operatorname{Mod}(\mathcal{R})$. We will still denote by $\rho_{!}$that functor. The functor $\rho_{*}: \operatorname{Mod}(\mathcal{R}) \rightarrow \operatorname{Mod}\left(\rho_{!} \mathcal{R}\right)$ is well defined too, in fact the morphism $\xi_{F} \in \operatorname{Hom}_{k_{X}}(\mathcal{R}, \mathcal{E} n d(F))$ defines a morphism in $\operatorname{Hom}_{k_{X_{s a}}}\left(\rho_{!} \mathcal{R}, \mathcal{E} n d\left(\rho_{*} F\right)\right)$. That follows from the chain of isomorphism

$$
\begin{aligned}
\operatorname{Hom}_{k_{X_{s a}}}\left(\rho_{!} \mathcal{R}, \mathcal{E} n d\left(\rho_{*} F\right)\right) & \simeq \operatorname{Hom}_{k_{X_{s a}}}\left(\rho_{!} \mathcal{R}, \rho_{*} \mathcal{E} n d(F)\right) \\
& \simeq \operatorname{Hom}_{k_{X}}\left(\rho^{-1} \rho_{!} \mathcal{R}, \mathcal{E} n d(F)\right) \\
& \simeq \operatorname{Hom}_{k_{X}}(\mathcal{R}, \mathcal{E} n d(F)) .
\end{aligned}
$$

We briefly summarize the properties of these functors:

- $\rho^{-1}$ commutes with $\otimes_{\mathcal{R}}^{L}, f^{-1}$ and $R f_{! !}$,

- $R \rho_{*}$ commutes with $R \mathcal{H}$ om ${ }_{\mathcal{R}}$ and $R f_{*}$,

- $\rho_{!}$commutes with $\otimes_{\mathcal{R}}^{L}$ and $f^{-1}$.

Finally we recall the following result (which has been proved in [7]) 
Proposition 3.2.1. Denote by $\widetilde{\mathcal{R}}$ the presheaf $U \rightarrow \Gamma(\bar{U} ; \mathcal{R})$, where $U \in \mathrm{Op}^{c}\left(X_{\text {sa }}\right)$. Suppose that $F$ is a presheaf of $\widetilde{\mathcal{R}}$-modules and denote by $F^{++}$the sheaf associated to $F$. Then $F^{++} \in \operatorname{Mod}\left(\rho_{!} \mathcal{R}\right)$.

Proof. Let $U \in \mathrm{Op}\left(X_{s a}\right)$, and let $r \in \Gamma(\bar{U} ; \mathcal{R})$. Then $r$ defines a morphism $\Gamma(\bar{V} ; \mathcal{R}) \otimes \Gamma(V ; F) \rightarrow \Gamma(V ; F)$ for each subanalytic $V \subset U$, hence an endomorphism of $\left.\left(F^{++}\right)\right|_{U_{X_{s a}}} \simeq\left(\left.F\right|_{U_{X_{s a}}}\right)^{++}$. This morphism defines a morphism of presheaves $\widetilde{\mathcal{R}} \rightarrow \mathcal{E} n d\left(F^{++}\right)$and $\widetilde{\mathcal{R}}^{++} \simeq \rho_{!} \mathcal{R}$ by Proposition 1.1.14. Then $F^{++} \in \operatorname{Mod}\left(\rho_{!} \mathcal{R}\right)$.

3.3 - Some examples of subanalytic sheaves.

From now on, the base field is $\mathrm{C}$. Let $M$ be a real analytic manifold. One denotes by $\mathcal{C}_{M}^{\infty}$ and $\mathcal{D} b_{M}$ the sheaves of $\mathcal{C}^{\infty}$ functions and Schwartz's distributions respectively, and by $\mathcal{D}_{M}$ the sheaf of finite order differential operators with analytic coefficients. As usual, given a sheaf $F$ on $M$, we set $D^{\prime} F=R \mathcal{H o m}\left(F, \mathrm{C}_{M}\right)$.

In [4] the author defined the functor

$$
T \mathcal{H o m}\left(\cdot, \mathcal{D} b_{M}\right): \operatorname{Mod}_{\mathrm{R}-\mathrm{c}}\left(\mathrm{C}_{M}\right)^{o p} \rightarrow \operatorname{Mod}\left(\mathcal{D}_{M}\right)
$$

in the following way: let $U$ be a subanalytic open subset of $M$ and $Z=M \backslash U$. Then the sheaf $T \mathcal{H} o m\left(\mathbb{C}_{U}, \mathcal{D} b_{M}\right)$ is defined by the exact sequence

$$
0 \rightarrow \Gamma_{Z} \mathcal{D} b_{M} \rightarrow \mathcal{D} b_{M} \rightarrow T \mathcal{H o m}\left(\mathbb{C}_{U}, \mathcal{D} b_{M}\right) \rightarrow 0
$$

This functor is exact and extends as a functor in the derived category, from $D_{\mathrm{R}-\mathrm{c}}^{b}\left(\mathrm{C}_{M}\right)$ to $D^{b}\left(\mathcal{D}_{M}\right)$. Moreover the sheaf $T \mathcal{H} \operatorname{Hom}\left(F, \mathcal{D} b_{M}\right)$ is soft for any $\mathbb{R}$ constructible sheaf $F$.

DEFinition 3.3.1. One denotes by $\mathcal{D} b_{M}^{t}$ the presheaf of tempered distributions on $M_{s a}$ defined as follows:

$$
U \mapsto \Gamma\left(M ; \mathcal{D} b_{M}\right) / \Gamma_{M \backslash U}\left(M ; \mathcal{D} b_{M}\right) .
$$

As a consequence of the Lojasievicz's inequalities [11], for $U, V \in$ $\in \mathrm{Op}\left(M_{s a}\right)$ the sequence

$$
0 \rightarrow \mathcal{D} b_{M}^{t}(U \cup V) \rightarrow \mathcal{D} b_{M}^{t}(U) \oplus \mathcal{D} b_{M}^{t}(V) \rightarrow \mathcal{D} b_{M}^{t}(U \cap V) \rightarrow 0
$$

is exact. Then $\mathcal{D} b_{M}^{t}$ is a sheaf on $M_{s a}$. Moreover it follows by definition that $\mathcal{D} b_{M}^{t}$ is quasi-injective. 
Definition 3.3.2. Let $Z$ be a closed subset of $M$. We denote by $\mathcal{I}_{M, Z}^{\infty}$ the sheaf of $\mathcal{C}^{\infty}$ functions on $M$ vanishing up to infinite order on $Z$.

Definition 3.3.3. A Whitney function on a closed subset $Z$ of $M$ is an indexed family $F=\left(F^{k}\right)_{k \in \mathbb{N}^{n}}$ consisting of continuous functions on $Z$ such that $\forall m \in \mathbb{N}, \forall k \in \mathbb{N}^{n},|k| \leq m, \forall x \in Z, \forall \varepsilon>0$ there exists a neighborhood $U$ of $x$ such that $\forall y, z \in U \cap Z$

$$
\left|F^{k}(z)-\sum_{|j+k| \leq m} \frac{(z-y)^{j}}{j !} F^{j+k}(y)\right| \leq \varepsilon d(y, z)^{m-|k|} .
$$

We denote by $W_{M, Z}^{\infty}$ the space of Whitney $\mathcal{C}^{\infty}$ functions on $Z$. We denote by $\mathcal{W}_{M, Z}^{\infty}$ the sheaf $U \mapsto W_{U, U \cap Z}^{\infty}$.

In [6] the authors defined the functor

$$
\cdot \stackrel{\mathrm{w}}{\otimes} \mathcal{C}_{M}^{\infty}: \operatorname{Mod}_{\mathrm{R}-\mathrm{c}}\left(\mathrm{C}_{M}\right) \rightarrow \operatorname{Mod}\left(\mathcal{D}_{M}\right)
$$

in the following way: let $U$ be a subanalytic open subset of $M$ and $Z=M \backslash U$. Then $\mathrm{C}_{U} \stackrel{\mathrm{w}}{\otimes} \mathcal{C}_{M}^{\infty}=\mathcal{I}_{M, Z}^{\infty}$, and $\mathrm{C}_{Z} \stackrel{\mathrm{w}}{\otimes} \mathcal{C}_{M}^{\infty}=\mathcal{W}_{M, Z}^{\infty}$. This functor is exact and extends as a functor in the derived category, from $D_{\mathbb{R}-c}^{b}\left(\mathbb{C}_{M}\right)$ to $D^{b}\left(\mathcal{D}_{M}\right)$. Moreover the sheaf $F \stackrel{\mathrm{w}}{\otimes} \mathcal{C}_{M}^{\infty}$ is soft for any $\mathbb{R}$-constructible sheaf $F$.

Definition 3.3.4. One denotes by $\mathcal{C}_{M}^{\infty, \mathrm{w}}$ the presheaf of Whitney $\mathcal{C}^{\infty}$ functions on $M_{\text {sa }}$ defined as follows:

$$
U \mapsto \Gamma\left(M ; \mathcal{H} o m\left(\mathbb{C}_{U}, \mathrm{C}_{M}\right) \stackrel{\mathrm{w}}{\otimes} \mathcal{C}_{M}^{\infty}\right) .
$$

As a consequence of a result of [12], for $U, V \in \mathrm{Op}\left(M_{s a}\right)$ the sequence

$$
0 \rightarrow \mathcal{C}_{M}^{\infty, \mathrm{w}}(U \cup V) \rightarrow \mathcal{C}_{M}^{\infty, \mathrm{w}}(U) \oplus \mathcal{C}_{M}^{\infty, \mathrm{w}}(V) \rightarrow \mathcal{C}_{M}^{\infty, \mathrm{w}}(U \cap V)
$$

is exact. Then $\mathcal{C}_{M}^{\infty, \mathrm{w}}$ is a sheaf on $M_{s a}$.

REMARK 3.3.5. Let us consider a locally cohomologically trivial (l.c.t.) subanalytic open subset, i.e. $U \in \mathrm{Op}\left(M_{s a}\right)$ satisfying $D^{\prime} \mathrm{C}_{U} \simeq \mathbb{C}_{\bar{U}}$ and $D^{\prime} \mathrm{C}_{\bar{U}} \simeq \mathbb{C}_{U}$. Thanks to the triangulation theorem one can prove that l.c.t. open subanalytic subsets form a basis for the topology of $M_{s a}$, and given a 
l.c.t. $U \in \mathrm{Op}\left(M_{s a}\right)$ we have

$$
\begin{aligned}
\Gamma\left(U ; \mathcal{C}_{M}^{\infty, \mathrm{w}}\right) & =\Gamma\left(M ; \mathcal{H o m}\left(\mathrm{C}_{U}, \mathrm{C}_{M}\right) \stackrel{\mathrm{w}}{\otimes} \mathcal{C}_{M}^{\infty}\right) \\
& \simeq \Gamma\left(M ; \mathrm{C}_{\bar{U}} \stackrel{\mathrm{w}}{\otimes} \mathcal{C}_{M}^{\infty}\right) \\
& =W_{M, \bar{U}}^{\infty} .
\end{aligned}
$$

Remark that $\Gamma\left(U ; \mathcal{D} b_{M}^{t}\right)$ and $\Gamma\left(U, \mathcal{C}_{M}^{\infty, \mathrm{w}}\right)$ are $\Gamma\left(\bar{U} ; \mathcal{D}_{M}\right)$-modules for each $U \in \mathrm{Op}\left(M_{s a}\right)$, hence applying Proposition 3.2.1 the sheaves $\mathcal{D} b_{M}^{t}$ and $\mathcal{C}_{M}^{\infty, \mathrm{w}}$ belong to $\operatorname{Mod}\left(\rho_{!} \mathcal{D}_{M}\right)$.

We have the following result

Proposition 3.3.6. For each $F \in D_{\mathbb{R}_{-c}}^{b}\left(C_{M}\right)$ one has the isomorphisms

$$
\begin{aligned}
\rho^{-1} \mathcal{H o m}\left(F, \mathcal{D} b_{M}^{t}\right) & \simeq T \mathcal{H} \operatorname{Hom}\left(F, \mathcal{D} b_{M}\right), \\
\rho^{-1} R \mathcal{H o m}\left(F, \mathcal{C}_{M}^{\infty, \mathrm{w}}\right) & \simeq D^{\prime} F \stackrel{\mathrm{w}}{\otimes} \mathcal{C}_{M}^{\infty} .
\end{aligned}
$$

Proof. We may reduce to the case $F=\mathrm{C}_{U}$ with $U \in \mathrm{Op}^{c}\left(M_{s a}\right)$. Let $V \in \mathrm{Op}^{c}\left(M_{s a}\right)$.

By definition of $T \mathcal{H}$ om we have $\Gamma\left(V ; T \mathcal{H}\right.$ om $\left.\left(\mathbb{C}_{U}, \mathcal{D} b_{M}\right)\right) \simeq$ $\simeq \Gamma\left(U \cap V ; \mathcal{D} b_{V}^{t}\right)$. Let us consider a subanalytic $W \subset \subset V$. The natural morphism $\Gamma\left(U \cap V ; \mathcal{D} b_{V}^{t}\right) \rightarrow \Gamma\left(U \cap W ; \mathcal{D} b_{M}^{t}\right)$ defines the morphism

$$
\varphi: \Gamma\left(U \cap V ; \mathcal{D} b_{V}^{t}\right) \rightarrow \lim _{W \subset C V} \Gamma\left(U \cap W ; \mathcal{D} b_{M}^{t}\right) \simeq \Gamma\left(V ; \rho^{-1} \Gamma_{U} \mathcal{D} b_{M}^{t}\right)
$$

Since the family $\left\{W \in \mathrm{Op}^{c}\left(M_{s a}\right) ; W \subset \subset V\right\}$ is a covering of $V$ and $T H \operatorname{Hom}\left(\mathrm{C}_{U}, \mathcal{D} b_{M}\right)$ is a sheaf $\varphi$ is an isomorphism.

To prove the second isomorphism we shall first prove the isomorphism

$$
\rho^{-1} \mathcal{H o m}\left(F, \mathcal{C}_{M}^{\infty, \mathrm{w}}\right) \simeq \mathcal{H o m}\left(F, \mathrm{C}_{M}\right) \stackrel{\mathrm{w}}{\otimes} \mathcal{C}_{M}^{\infty}
$$

for $F \in \operatorname{Mod}_{\mathbb{R}-c}\left(C_{M}\right)$. We may reduce to the case $F=\mathrm{C}_{U}$ with $U$ l.c.t. and subanalytic. Let $V \in \mathrm{Op}^{c}\left(M_{s a}\right)$ such that $V$ and $U \cap V$ are 1.c.t. and let us consider the family $\mathcal{T}=\left\{W \in \mathrm{Op}^{c}\left(M_{s a}\right)\right.$ l.c.t.; $W \subset \subset V, W \cap U$ l.c.t. $\}$. The natural morphism $\psi: \Gamma\left(V ; \mathrm{C}_{\bar{U}} \otimes \mathcal{C}_{M}^{\infty}\right) \simeq W_{V, V \cap \bar{U}}^{\infty} \rightarrow W_{M, \bar{U} \cap W}^{\infty} \simeq$ $\Gamma\left(M ; \mathrm{C}_{\overline{U \cap W}} \stackrel{\mathrm{w}}{\otimes} \mathcal{C}_{M}^{\infty}\right)$ defines the morphism

$$
\psi: \Gamma\left(V ; \mathbb{C}_{\bar{U}} \stackrel{\mathrm{w}}{\otimes} \mathcal{C}_{M}^{\infty}\right) \rightarrow \lim _{W \in \mathcal{T}} \Gamma\left(M ; \mathrm{C}_{\overline{U \cap W}} \stackrel{\mathrm{w}}{\otimes} \mathcal{C}_{M}^{\infty}\right) \simeq \Gamma\left(V ; \rho^{-1} \Gamma_{U} \mathcal{C}_{M}^{\infty, \mathrm{w}}\right),
$$


where the second isomorphism follows since the family $\mathcal{T}$ is cofinal in $\left\{W \in \mathrm{Op}^{c}\left(M_{s a}\right) ; W \subset \subset V\right\}$. Moreover we have the isomorphism

$$
\lim _{W \in \mathcal{T}} \Gamma\left(M ; \mathrm{C}_{\overline{U \cap W}} \stackrel{\mathrm{w}}{\otimes} \mathcal{C}_{M}^{\infty}\right) \simeq{\underset{W \in \mathcal{T}}{\leftrightarrows}}_{\lim } \Gamma\left(W ; \mathrm{C}_{\bar{U}} \stackrel{\mathrm{w}}{\otimes} \mathcal{C}_{M}^{\infty}\right) .
$$

Since the family $\mathcal{T}$ is a covering of $V$ and $\mathrm{C}_{\bar{U}} \stackrel{\mathrm{w}}{\otimes} \mathcal{C}_{M}^{\infty}$ is a sheaf $\psi$ is an isomorphism. Hence we get the desired isomorphism.

Now let $F \in D_{\mathbb{R}-c}^{b}\left(C_{M}\right)$. We have the chain of morphisms

$$
\begin{aligned}
D^{\prime} F \stackrel{\mathrm{w}}{\otimes} \mathcal{C}_{M}^{\infty} & \simeq \underset{F^{\prime} \rightarrow F}{\lim } \operatorname{Hom}\left(F^{\prime}, \mathrm{C}_{M}\right) \stackrel{\mathrm{w}}{\otimes} \mathcal{C}_{M}^{\infty} \\
& \simeq \underset{F^{\prime} \rightarrow F}{\lim } \rho^{-1} \mathcal{H o m}\left(F^{\prime}, \mathcal{C}_{M}^{\infty, \mathrm{w}}\right) \\
& \simeq \rho^{-1} R \mathcal{H o m}\left(F, \mathcal{C}_{M}^{\infty, \mathrm{w}}\right),
\end{aligned}
$$

where $F^{\prime} \rightarrow F$ ranges to the family of qis. By Theorem 2.1.2 we may suppose $F^{\prime} \in K^{b}\left(\operatorname{Mod}_{\mathbb{R}-c}\left(k_{M}\right)\right)$, then we can restrict to $F^{\prime}=\mathrm{C}_{U}$, $U \in \mathrm{Op}^{c}\left(M_{s a}\right)$ l.c.t. and the second isomorphism follows from (3.1). The third isomorphism follows since $\rho^{-1}$ is exact.

REMARK 3.3.7. As a consequence of Proposition 3.3.6, given $U \in$ $\mathrm{Op}\left(M_{s a}\right)$ we have $R \Gamma\left(U ; \mathcal{C}_{M}^{\infty, \mathrm{w}}\right) \simeq R \Gamma\left(M ; D^{\prime} \mathrm{C}_{U} \stackrel{\mathrm{w}}{\otimes} \mathcal{C}_{M}^{\infty}\right)$. In particular when $U$ is l.c.t. $R \Gamma\left(U ; \mathcal{C}_{M}^{\infty, \mathrm{w}}\right)$ is concentrated in degree zero since $\mathrm{C}_{\bar{U}} \stackrel{\mathrm{w}}{\otimes} \mathcal{C}_{M}^{\infty}$ is soft.

Now let $X$ be a complex manifold, $X_{\mathrm{R}}$ the underlying real analytic manifold and $\bar{X}$ the complex conjugate manifold. One denotes by $\mathcal{O}_{X}^{t}$ and $\mathcal{O}_{X}^{\mathrm{W}}$ the sheaves of tempered and Whitney holomorphic functions respectively which are defined as follows:

$$
\begin{aligned}
\mathcal{O}_{X}^{t} & :=R \mathcal{H o m} \operatorname{lo}_{\rho_{X}}\left(\rho_{!} \mathcal{O}_{\bar{X}}, \mathcal{D} b_{X_{\mathbb{R}}^{t}}\right) \\
\mathcal{O}_{X}^{\mathrm{W}} & :=R \mathcal{H o m} \rho_{\rho_{1} \mathcal{D}_{X}}\left(\rho_{!} \mathcal{O}_{\bar{X}}, \mathcal{C}_{X_{\mathrm{R}}}^{\infty, \mathrm{w}}\right) .
\end{aligned}
$$

By definition, $\mathcal{O}_{X}^{t}$ and $\mathcal{O}_{X}^{\mathrm{W}}$ belong to $D^{b}\left(\rho_{!} \mathcal{D}_{X}\right)$. The relation with the functors of temperate and formal cohomology is given by the following result

Proposition 3.3.8. For each $F \in D_{\mathbb{R}-\mathrm{c}}^{b}\left(\mathbb{C}_{X}\right)$ one has the isomorphisms

$$
\begin{aligned}
& \rho^{-1} R \mathcal{H} \operatorname{Hom}\left(F, \mathcal{O}_{X}^{t}\right) \simeq T \mathcal{H o m}\left(F, \mathcal{O}_{X}\right), \\
& \rho^{-1} R \mathcal{H} \operatorname{Hom}\left(F, \mathcal{O}_{X}^{\mathrm{w}}\right) \simeq D^{\prime} F \stackrel{\mathrm{w}}{\otimes} \mathcal{O}_{X} .
\end{aligned}
$$


Proof. We have the chain of isomorphisms

$$
\begin{aligned}
\rho^{-1} R \mathcal{H o m}\left(F, \mathcal{O}_{X}^{t}\right) & \simeq \rho^{-1} R \mathcal{H o m}\left(F, R \mathcal{H o m} \operatorname{pom}_{\rho_{X}}\left(\rho_{!} \mathcal{O}_{\bar{X}}, \mathcal{D} b_{X_{\mathrm{R}}}^{t}\right)\right) \\
& \simeq \rho^{-1} R \mathcal{H} \operatorname{Hom}_{\rho_{!} \mathcal{D}_{X}}\left(\rho_{!} \mathcal{O}_{\bar{X}}, R \mathcal{H o m}\left(F, \mathcal{D} b_{X_{\mathbb{R}}}^{t}\right)\right) \\
& \simeq R \mathcal{H o m} \bar{D}_{\mathcal{D}_{X}}\left(\mathcal{O}_{\bar{X}}, \rho^{-1} R \mathcal{H o m}\left(F, \mathcal{D} b_{X_{\mathrm{R}}}\right)\right) \\
& \simeq R \mathcal{H o m}\left(\mathcal{O}_{\bar{X}}, T \mathcal{H o m}\left(F, \mathcal{D} b_{X_{\mathrm{R}}}^{t}\right)\right) \\
& \simeq \operatorname{THom}\left(F, \mathcal{O}_{X}\right) .
\end{aligned}
$$

The proof of $\rho^{-1} R \mathcal{H o m}\left(F, \mathcal{O}_{X}^{\mathrm{W}}\right) \simeq D^{\prime} F \stackrel{\mathrm{w}}{\otimes} \mathcal{O}_{X}$ is similar.

\section{A. Appendix.}

A.1 - Review on subanalytic sets.

We recall briefly some properties of subanalytic subsets. References are made to [1] and [10]. Let $X$ be a real analytic manifold.

Definition A.1.1. Let $A$ be a subset of $X$.

(i) $A$ is said to be semi-analytic if it is locally analytic, i.e. each $x \in A$ has a neighborhood $U_{x}$ such that $X \cap U_{x}=\cup_{i \in I} \cap_{j \in J} X_{i j}$, where $I, J$ are finite sets and either $X_{i j}=\left\{y \in U_{x} ; f_{i j}>0\right\}$ or $X_{i j}=\left\{y \in U_{x} ; f_{i j}=0\right\}$ for some analytic function $f_{i j}$.

(ii) $A$ is said to be subanalytic if it is locally a projection of a relatively compact semi-analytic subset, i.e. each $x \in A$ has a neighborhood $U$ such that there exists a real analytic manifold $Y$ and a relatively compact semi-analytic subset $A^{\prime} \subset X \times Y$ satisfying $X \cap U=\pi\left(A^{\prime}\right)$, where $\pi: X \times Y \rightarrow X$ denotes the projection.

(iii) Let $Y$ be a real analytic manifold. A continuous map $f: X \rightarrow Y$ is subanalytic if its graph is subanalytic in $X \times Y$.

Let us recall some result on subanalytic subsets.

Proposition A.1.2. Let $A, B$ be subanalytic subsets of $X$. Then $A \cup B$, $A \cap B, \bar{A}, \partial A$ and $A \backslash B$ are subanalytic.

Proposition A.1.3. Let $A$ be a subanalytic subsets of $X$. Then the connected components of $A$ are locally finite. 
Proposition A.1.4. Let $f: X \rightarrow Y$ be a subanalytic map. Let $A$ be a relatively compact subanalytic subset of $X$. Then $f(A)$ is subanalytic.

Definition A.1.5. A simplicial complex $(K, \Delta)$ is the data consisting of a set $K$ and $a$ set $\Delta$ of subsets of $K$ satisfying the following axioms:

S1 any $\sigma \in \Delta$ is a finite and non-empty subset of $K$,

S2 if $\tau$ is a non-empty subset of an element $\sigma$ of $\Delta$, then $\tau$ belongs to $\Delta$,

S3 for any $p \in K,\{p\}$ belongs to $\Delta$,

$\mathrm{S} 4$ for any $p \in K$, the set $\{\sigma \in \Delta ; p \in \sigma\}$ is finite.

If $(K, \Delta)$ is a simplicial complex, an element of $K$ is called a vertex. Let $\mathbb{R}^{K}$ be the set of maps from $K$ to $\mathbb{R}$ equipped with the product topology. To $\sigma \in \Delta$ one associate $|\sigma| \subset \mathbb{R}^{K}$ as follows:

$$
|\sigma|=\left\{x \in \mathbb{R}^{K} ; x(p)=0 \text { for } p \notin \sigma, x(p)>0 \text { for } p \in \sigma \text { and } \sum_{p} x(p)=1\right\} .
$$

As usual we set:

$$
\begin{gathered}
|K|=\bigcup_{\sigma \in \Delta}|\sigma|, \\
U(\sigma)=\bigcup_{\tau \in \Delta, \tau \supset \sigma}|\tau|,
\end{gathered}
$$

and for $x \in|K|$ :

$$
U(x)=U(\sigma(x)),
$$

where $\sigma(x)$ is the unique simplex such that $x \in|\sigma|$.

Theorem A.1.6. Let $X=\bigsqcup_{i \in I} X_{i}$ be a locally finite partition of $X$ consisting of subanalytic subsets. Then there exists a simplicial complex $(K, \Delta)$ and a subanalytic homeomorphism $\psi:|K| \stackrel{\sim}{\rightarrow} X$ such that

(i) for any $\sigma \in \Delta, \psi(|\sigma|)$ is a subanalytic submanifold of $X$,

(ii) for any $\sigma \in \Delta$ there exists $i \in I$ such that $\psi(|\sigma|) \subset X_{i}$.

\section{A.2 - Sheaves on Grothendieck topologies.}

We recall the definition of a Grothendieck topology. We will not treat the most general case, for which we refer to [8]. We will follow the presentation of [14] and [7]. 
Let $\mathcal{C}$ be a category admitting finite products and fiber products, and given $U \in \mathcal{C}$, denote by $\mathcal{C}_{U}$ the category of arrows $V \rightarrow U$. Given a morphism $V \rightarrow U$ and $S \subset \mathrm{Ob}\left(\mathcal{C}_{U}\right)$, one denotes by $V \times_{U} S \subset \mathrm{Ob}\left(\mathcal{C}_{V}\right)$ the subset defined by $\left\{V \times_{U} W \rightarrow V ; W \in S\right\}$.

Definition A.2.1. If, $S_{1}, S_{2} \subset \mathrm{Ob}\left(\mathcal{C}_{U}\right)$, one says that $S_{1}$ is a refinement of $S_{2}\left(S_{1} \preceq S_{2}\right.$ for short) if any $V_{1} \rightarrow U$ in $S_{1}$ factorizes as $V_{1} \rightarrow V_{2} \rightarrow U$ with $V_{2} \rightarrow U \in S_{2}$.

Definition A.2.2. A Grothendieck topology on $\mathcal{C}$ associates to each $U \in \mathcal{C}$ a family $\operatorname{Cov}(U) \subset \mathrm{Ob}\left(C_{U}\right)$ satisfying the following axioms:

$G T 1\{U \stackrel{i d}{\rightarrow} U\} \in \operatorname{Cov}(U)$,

GT2 if $\operatorname{Cov}(U) \ni S_{1} \preceq S_{2} \subset \mathrm{Ob}\left(\mathcal{C}_{U}\right)$, then $S_{2} \in \operatorname{Cov}(U)$,

$G T$ 3 if $S \in \operatorname{Cov}(U)$, then for each $V \rightarrow U, V \times{ }_{U} S \in \operatorname{Cov}(V)$,

GT4 if $S_{1}, S_{2} \subset \mathrm{Ob}\left(\mathcal{C}_{U}\right), S_{1} \in \operatorname{Cov}(U)$ and $V \times_{U} S_{2} \in \operatorname{Cov}(V)$, then $S_{2} \in$ $\operatorname{Cov}(U)$.

An object $S \in \operatorname{Cov}(U)$ is called a covering of $U$.

Definition A.2.3. A site $X$ is a category $\mathcal{C}_{X}$ endowed with a Grothendieck topology.

Let $\mathcal{C}_{X}$ and $\mathcal{C}_{Y}$ be two categories admitting finite products and fiber products. A functor of sites $f: X \rightarrow Y$ is a functor $f^{t}: \mathcal{C}_{Y} \rightarrow C_{X}$ which commutes with fiber products and such that if $U \in \mathcal{C}_{Y}$ and $S \in \operatorname{Cov}(U)$, then $f^{t}(S) \in \operatorname{Cov}\left(f^{t}(U)\right)$.

Now let $k$ be a field.

Definition A.2.4. Let $X$ be a site. A presheaf of $k$-modules on $X$ is a functor $\mathcal{C}_{X}^{o p} \rightarrow \operatorname{Mod}(k)$.

One denotes by $\operatorname{Psh}\left(k_{X}\right)$ the abelian category of presheaves of $k$-modules on $X$. Let $F \in \operatorname{Psh}\left(k_{X}\right)$, let $U \in \mathcal{C}_{X}$ and consider $V \rightarrow U \in \mathcal{C}_{U}$. The restriction morphism $F(U) \rightarrow F(V)$ is denoted by $\left.s \mapsto s\right|_{U}$.

Let $F$ be a presheaf of $k$-modules on $X$ and let $S \subset \mathrm{Ob}\left(\mathcal{C}_{U}\right)$. One defines

$$
F(S)=\operatorname{ker}\left(\prod_{V \in S} F(V) \rightrightarrows \prod_{V^{\prime}, V^{\prime \prime} \in S} F\left(V^{\prime} \times_{U} V^{\prime \prime}\right)\right)
$$


Definition A.2.5. A presheaf $F$ of $k$-modules on $X$ is a separated presheaf (resp. a sheaf) if for each $U \in \mathcal{C}_{X}$ and each $S \in \operatorname{Cov}(U)$ the morphism $F(U) \rightarrow F(S)$ is a monomorphism (resp. an isomorphism).

One denotes by $\operatorname{Mod}\left(k_{X}\right)$ the category of sheaves of $k$-modules on $X$. We set for short $\operatorname{Hom}_{k_{X}}$ instead of $\operatorname{Hom}_{\operatorname{Mod}\left(k_{X}\right)}$.

We recall the construction of a sheaf associated to a presheaf. The relation " $\preceq$ " defines a preorder on $\operatorname{Cov}(U), U \in \mathcal{C}_{X}$. Let $F \in \operatorname{Psh}\left(k_{X}\right)$, one defines the functor $(\cdot)^{+}: \operatorname{Psh}\left(k_{X}\right) \rightarrow \operatorname{Psh}\left(k_{X}\right)$ in the following way. For each $U \in \mathcal{C}_{X}$

$$
F^{+}(U)=\underset{S \in \operatorname{Cov}(U)}{\lim } F(S)
$$

Theorem A.2.6. (i) The functor $(\cdot)^{+}: \operatorname{Psh}\left(k_{X}\right) \rightarrow \operatorname{Psh}\left(k_{X}\right)$ is left exact,

(ii) if $F \in \operatorname{Psh}\left(k_{X}\right)$, then $F^{+}$is separated,

(iii) if $F \in \operatorname{Psh}\left(k_{X}\right)$ is separated, then $F^{+} \in \operatorname{Mod}\left(k_{X}\right)$,

(iv) the functor $(\cdot)^{++}: \operatorname{Psh}\left(k_{X}\right) \rightarrow \operatorname{Mod}\left(k_{X}\right)$ is exact,

(v) let $F \in \operatorname{Psh}\left(k_{X}\right)$ and $G \in \operatorname{Mod}\left(k_{X}\right)$, one has the adjunction formula:

$$
\operatorname{Hom}_{P s h\left(k_{X}\right)}(F, l G) \simeq \operatorname{Hom}_{k_{X}}\left(F^{++}, G\right),
$$

where $\mathrm{l}$ denotes the embedding functor.

Let $F \in \operatorname{Psh}\left(k_{X}\right)$, the sheaf $F^{++}$is called the sheaf associated to $F$.

Proposition A.2.7. Let $F, G \in \operatorname{Mod}\left(k_{X}\right)$. A morphism $\varphi \in \operatorname{Hom}_{k_{X}}(F, G)$ is an epimorphism if and only if for each $U \in \mathcal{C}_{X}$ there exists $\left\{U_{i}\right\}_{i \in I} \in \operatorname{Cov}(U)$ such that for each $s \in G(U)$ there exists $t_{i} \in F\left(U_{i}\right)$ such that $\varphi\left(t_{i}\right)=\left.s\right|_{U_{i}}$ for each $i$.

Let $f: X \rightarrow Y$ be a morphism of sites. Let $F \in \operatorname{Psh}\left(k_{X}\right)$ and $G \in$ $\in \operatorname{Psh}\left(k_{Y}\right)$. One defines the functors

$$
\begin{aligned}
& f_{*}: \operatorname{Psh}\left(k_{X}\right) \rightarrow \operatorname{Psh}\left(k_{Y}\right) \\
& f^{\leftarrow}: \operatorname{Psh}\left(k_{Y}\right) \rightarrow \operatorname{Psh}\left(k_{X}\right)
\end{aligned}
$$


in the following way: let $U \in \mathcal{C}_{X}$ and $V \in \mathcal{C}_{Y}$, then

$$
\begin{gathered}
f_{*} F(V)=F\left(f^{t}(V)\right) \\
f^{\leftarrow} F(U)=\underset{U \rightarrow f^{t}(W)}{\longrightarrow} G(W),
\end{gathered}
$$

where $W \in \mathcal{C}_{Y}$.

Definition A.2.8. Let $f: X \rightarrow Y$ be a functor of sites

(i) the functor of direct image $f_{*}: \operatorname{Mod}\left(k_{X}\right) \rightarrow \operatorname{Mod}\left(k_{Y}\right)$ is the functor induced by (A.1),

(ii) the functor of inverse image $f^{-1}: \operatorname{Mod}\left(k_{Y}\right) \rightarrow \operatorname{Mod}\left(k_{X}\right)$ is defined by $f^{-1}=\left(f^{\leftarrow}(\cdot)\right)^{++}$.

Proposition A.2.9. (i) The functor $f_{*}$ is left exact and commutes with $\underset{\longleftarrow}{\mathrm{lim}}$

(ii) the functor $f^{-1}$ is exact and commutes with $\underline{\mathrm{lim}}$,

(iii) $\left(f^{-1}, f_{*}\right)$ is a pair of adjoint functors.

Definition A.2.10. Let $X$ be a site and let $F, G \in \operatorname{Mod}\left(k_{X}\right)$.

(i) One denotes by $\mathcal{H o m}(F, G)$ the sheaf $U \mapsto \operatorname{Hom}_{k_{U}}\left(\left.F\right|_{U},\left.G\right|_{U}\right)$,

(ii) one denotes by $F \otimes G$ the sheaf associated to the presheaf $U \mapsto F(U) \otimes G(U)$.

Proposition A.2.11. Let $F \in \operatorname{Mod}\left(k_{X}\right), G, G^{\prime} \in \operatorname{Mod}\left(k_{Y}\right)$.

(i) $\mathcal{H o m}_{k_{Y}}\left(G, f_{*} F\right) \simeq f_{*} \mathcal{H o m}_{k_{X}}\left(f^{-1} G, F\right)$,

(ii) $f^{-1}\left(G \otimes G^{\prime}\right) \simeq f^{-1} G \otimes f^{-1} G^{\prime}$.

\section{REFERENCES}

[1] E. Bierstorne - D. Milmann, Semianalytic and subanalytic sets, Publ. I.H.E.S., 67 (1988), pp. 5-42.

[2] S. I. Gelfand - Yu. I. Manin, Methods of homological algebra, SpringerVerlag, Berlin (1996).

[3] R. Godement, Topologie algébrique et théorie des faisceaux, Hermann, Paris (1958). 
[4] M. Kashiwara, The Riemann-Hilbert problem for holonomic systems, Publ. RIMS, Kyoto Univ., 20 (1984), pp. 319-365.

[5] M. Kashiwara - P. SchapiRa, Sheaves on manifolds, Grundlehren der Math. 292, Springer-Verlag, Berlin (1990).

[6] M. KASHIWARA - P. SCHAPIRA, Moderate and formal cohomology associated with constructible sheaves, Mémoires Soc. Math. France, 64 (1996).

[7] M. KaShIWARA - P. SchaPIRA, Ind-sheaves, Astérisque, 271 (2001).

[8] M. Kashiwara - P. SchapiRa, Categories and sheaves, Grundlehren der Math., 332, Springer-Verlag, Berlin (2005).

[9] B. KeLler, Derived categories and their uses, Handbook of algebra vol. 1 pp. 671-701 North Holland, Amsterdam (1996).

[10] S. LoJACIEWICZ, Sur la géométrie semi- et sous-analytique, Ann. Inst. Fourier, 43 (1993), pp. 1575-1595.

[11] S. Lojaciewicz, Sur le problème de la division, Studia Mathematica, 8 (1959) pp. 87-136.

[12] B. Malgrange, Ideals of differentiable functions, Tata Institute, Oxford University Press (1967).

[13] L. Prelli, Sheaves on subanalytic sites, Phd Thesis, Universities of Padova and Paris 6 (2006).

[14] G. TAMmE, Introduction to étale cohomology, Universitext Springer-Verlag, Berlin (1994).

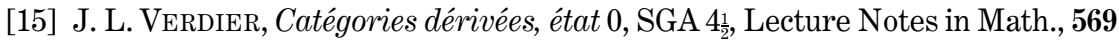
Springer-Verlag, Berlin (1977).

[16] SGA4: Sém. Géom. Algébrique du Bois-Marie by M. Artin, A. Grothendieck, J. L. Verdier, Lecture Notes in Math. 269 Springer-Verlag, Berlin (1972).

Manoscritto pervenuto in redazione il 14 maggio 2007. 